\title{
Experimental interpretation of adequate weight-metric combination for dynamic user-based collaborative filtering
}

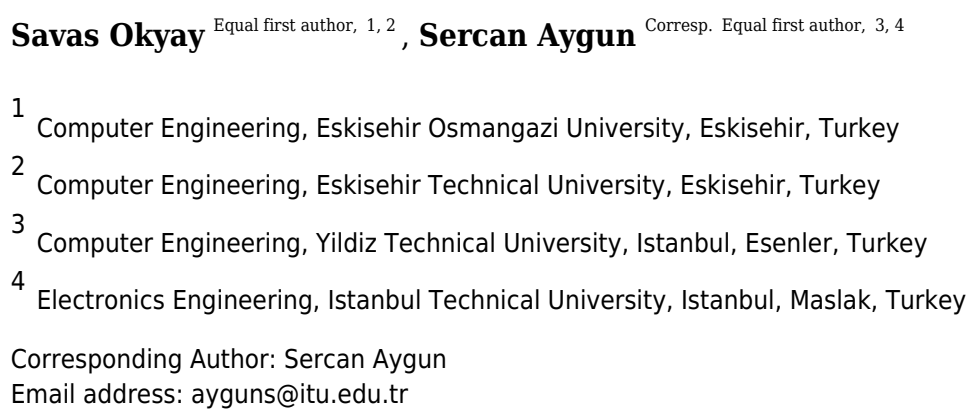

Recommender systems include a broad scope of applications and are associated with subjective preferences, indicating variations in recommendations. As a field of data science and machine learning, recommender systems require both statistical perspectives and sufficient performance monitoring. In this paper, we propose diversified similarity measurements by observing recommendation performance using generic metrics. Considering user-based collaborative filtering, the probability of an item being preferred by any user is measured. Having examined the best neighbor counts, we verified the test item bias phenomenon for similarity equations. Because of the statistical parameters used for computing in a global scope, there is implicit information in the literature, whether those parameters comprise the focal point user data statically. Regarding each dynamic prediction, user-wise parameters are expected to be generated at runtime by excluding the item of interest. This yields reliable results and is more compatible with real-time systems. Furthermore, we underline the effect of significance weighting by examining the similarities between a user of interest and its neighbors. Overall, this study uniquely combines significance weighting and test-item bias mitigation by inspecting the fine-tuned neighborhood. Consequently, the results reveal adequate similarity weight and performance metric combinations. The source code of our architecture is available at https://codeocean.com/capsule/1427708/tree/v1. 
1 Experimental Interpretation of Adequate Weight-Metric

2 Combination for Dynamic User-Based Collaborative

3 Filtering

4

Savas Okyay ${ }^{1,2}$, Sercan Aygun ${ }^{3,4}$

${ }^{1}$ Computer Engineering Dept., Eskisehir Osmangazi University, Eskisehir, Turkey

${ }^{2}$ Computer Engineering Dept., Eskisehir Technical University, Eskisehir, Turkey

${ }^{3}$ Computer Engineering Dept., Yildiz Technical University, Istanbul, Turkey

${ }^{4}$ Electronics Engineering Dept., Istanbul Technical University, Istanbul, Turkey

*Authors have equal contributions, and names are given alphabetically.

Corresponding Author:

Sercan Aygun ${ }^{4}$

Davutpasa, Istanbul, Esenler, 34220, Turkey

Email address: ayguns@itu.edu.tr

\section{Abstract}

Recommender systems include a broad scope of applications and are associated with subjective preferences, indicating variations in recommendations. As a field of data science and machine learning, recommender systems require both statistical perspectives and sufficient performance monitoring. In this paper, we propose diversified similarity measurements by observing recommendation performance using generic metrics. Considering user-based collaborative filtering, the probability of an item being preferred by any user is measured. Having examined the best neighbor counts, we verified the test item bias phenomenon for similarity equations. Because of the statistical parameters used for computing in a global scope, there is implicit information in the literature, whether those parameters comprise the focal point user data statically. Regarding each dynamic prediction, user-wise parameters are expected to be generated at runtime by excluding the item of interest. This yields reliable results and is more compatible with real-time systems. Furthermore, we underline the effect of significance weighting by examining the similarities between a user of interest and its neighbors. Overall, this study uniquely combines significance weighting and test-item bias mitigation by inspecting the finetuned neighborhood. Consequently, the results reveal adequate similarity weight and performance metric combinations. The source code of our architecture is available at https://codeocean.com/capsule/1427708/tree/v1.

\section{Introduction}


39 Recommender systems (RS) are utilized in various applications, and users interact with them on

40 a range of application-specific platforms. Personal data and previous activities are combined to

41 understand a user's taste. Any recommendation of items on a platform can be provided.

42 Considering both online and offline applications, a stable architecture is essential for machine

43 learning and for promoting the business of any platform. Recommender systems have been

44 implemented on various platforms, including social media [1], healthcare [2], journals [3], music

45 [4], [5], suggestion systems, and movie recommendation frameworks [6]-[8]. Recommender

46 systems focus on analyzing preferences and deciding the prospective action of a user. A person

47 performs activities (such as passing remarks, leaving comments, giving rates, liking, or disliking

48 products) on a specific application because these activities are all logged into a database. Movie-

49 based RS has been the focus of many data scientists for two significant reasons. First, scientific

50 datasets such as MovieLens [9] and Netflix [10] are readily available and easy to use. Second, an

51 overall RS architecture has been established that is entirely compatible with additional user and

52 item features, enabling scientists to measure two well-known phenomena in collaborative

53 filtering (CF): (i) user-based similarity and (ii) item-based similarity.

54 This study aims to measure the effect of correlation adjustment. Considering the literature, there are many reported RS implementations; however, it is unclear how the inclusion or exclusion of a test item is determined during statistical parameter computations. Similarity calculations between two users require analytical computations, such as the mean and median. Theoretical studies may set statistical arguments as global parameters to encapsulate computations for all upcoming test attempts concerning time complexity and memory management. Although setting parameters globally is computation-friendly, if all statistical primitives are set in this wide scope, test items become less dependent on the related test attempts. Hence, the expected recommendation may be slightly false. Considering real-time applications, the rating value of the recommended item is unknown. In this study, the dynamic effect of the item-of-interest (IOI) is examined to demonstrate the difference between theoretical and real-time performance. In addition, the co-rated item count (CIC) between the user-of-interest and its neighbors is utilized to revise the calculated similarity weight for further constant multiplications [11]-[16]. Thus, the correlation between users is connected to the commonly rated item counts. We refer to this multiplication as the CIC-based significance weighting $(S W)$ method by demonstrating its performance. We interpret the efficiency of the IOI and $S W$ conditions based on four different similarity equations, including Pearson similarity, median-based robust correlation, cosine similarity, and Jaccard similarity.

72 In general, studies have focused on finding ways to increase the efficiency of RS. The closer the forecast is to the user preference obtained, the more accurate the system design is. However, the performance metrics of a system can be more than a single prediction accuracy. In this study, we examined previously proposed similarity equations and performance metrics. The research constructs a perspective on how to connect user similarity measurements to an enlarged number

77 of performance metrics, including those from other disciplines. Schröder et al. propose the utilization of relatively less known metrics such as informedness, markedness, and Matthews 
79

80

81

82

83

84

85

86

87

88

89

90

91

92

93

94

95

96

97

98

99

100

101

102

103

104

105

106

107

108

109

110

111

112

113

114

correlation because they are superior to precision, recall, and F1-measure [17]. Schröder et al. acknowledge that these performance metrics are suitable for determining the top- $n$ recommendation in e-commerce applications; therefore, we evaluate the performance of these metrics compared to the well-known ones.

Previous RS implementations have either a relatively small set of metrics for testing [18]-[21] or a limited range of specific parameters, such as the best neighborhood [11], [22]-[26]. Any user is provided with a recommendation by examining the closest neighbors who have the same tendencies for the related IOI. Instead of setting the best neighbor count $(B N C)$ to a constant value, the neighborhood should be appropriately determined. Therefore, we parameterize the number of neighbors, s.t., using $\varepsilon$ step size between the least neighbor count $(L N C)$ and most neighbor count $(M N C)$.

A comprehensive back-end software architecture has been developed in this study. Our framework ${ }^{1}$ is an adaptive tool that enables the test environment to capture the general behavior of high-density datasets with an adjusted $\varepsilon$.

To the best of our knowledge, no previous studies on RS have extensively focused on an adequate combination of similarity measurements and performance metrics. Overall, the following highlights are presented in the scope of this study.

- We construct an RS framework that highlights the possible pitfalls and enhancements in RS architectural designs. Therefore, the following two perspectives are applied to the similarity equations.

- The first perspective underlines the dynamicity principles of real-time systems by excluding the $I O I$, known as no item-of-interest (nIOI).

- The second perspective emphasizes the results of the utilization of significant weights. Considering the $S W$ method, the more common the rating counts from neighbors observed are, the more significant the weights.

- The $B N C$ is analyzed and determined experimentally considering a number of performance metrics.

- Extensive tests are applied to popular MovieLens releases with randomized trials of separate runs.

- Considering the evaluation, relatively less known performance metrics such as informedness, markedness, and Matthews correlation are examined comprehensively. In addition, established metrics such as precision, sensitivity, specificity, F1-measure, fallout, miss rate, etc., including error metrics, are compared. These prediction-oriented metrics have been extensively demonstrated with notable outcomes.

- Prevalence threshold and threat score, which are frequently practiced in other disciplines, are analyzed in the context of RS.

${ }^{1}$ Open-source code information is given in the Acknowledgments. Any dataset can be analyzed as long as it meets the requirement of user $\times$ item matrix format. 
115

116

117

118

119

120

121

122

123

124

125

126

127

128

129

130

131

132

133

134

135

136

137

138

139

140

141

142

143

144

145

146

147

148

149

150

151

152

153

154

- Finally, the heat-map tables for the top-performing BNCs connected to the adequate weightmetric combinations are presented.

The brief of the overall paper structure and each subsection content are visually presented in Fig. 1. The remainder of this study is organized as follows. The materials and methods are provided in Section II, wherein the nomenclature and dataset details are presented. In addition, similarity equations and performance metrics used throughout this study are presented in the same section. Section III includes the details of the computation environment and preliminary selection of topperforming neighbors. This is followed by the extensive results in Section IV. In the last section, the conclusion and recommendations for future research are presented.

\section{Materials \& Methods}

This section describes the dataset used and the methods applied. First, the technical details of MovieLens releases are provided. Thereafter, the touchstone similarity equations and the modifications, considering the $n I O I$ phenomenon and $S W$, are discussed. Finally, the performance metrics implemented in the proposed RS framework are presented. The symbols and abbreviations used throughout this study are listed in Table 1.

\section{A. The MovieLens}

Considering the current RS applications, the basic practical structure of data commonly has a user $\times$ item matrix format. One of the frequently trained scientific datasets is MovieLens [27] which has several releases based on size and additional content.

Considering Table 2, the main types of MovieLens can be reviewed depending on the rating size. For example, ML100K has 100,000 clicks. The MovieLens dataset is upgraded several times for the expanded types and for the versions of previous releases. For instance, the ML100K type has various releases, such as one that includes one to five ratings with only decimal values. The latest ML100K version consists of 0.5 steps between ratings, including half stars. However, this version is not recommended for shared research results because it is a developing dataset.

Several previous studies focused on the tried-and-trusted original ML100K release, which is a pioneering collection and has considerably efficient runtime performance. Considering the scope of this study, we utilize this original release, which includes only full stars. Additionally, we encapsulated extensive experiments of ML1M to maintain full-star rating scaling parallelism with the ML100K. Therefore, we comparatively present the results related to the original ML100K and ML1M.

In this section, preliminary dataset analyses are presented. To validate the methods used in the following sections, residual checks on user-based statistical arguments and item-based independence analyses were performed as follows.

\section{1) Checking residuals on user-based statistical arguments}

The dynamicity effect of user-based statistical arguments (such as the mean and median) is discussed in this subsection. As static and dynamic approaches are the main focus, a visualization of their residual analysis on the utilization of the arguments is presented. Therefore, 
155 the rating history of each user was examined based on the statistical observations. Considering

156

157

158

159

160

161

162

163

164

165

166

167

168

169

170

171

172

173

174

175

176

177

178

179

180

181

182

183

184

185

186

187

188

189

190

191

192

193

194 any user, it is observed that the statistical values of all the ratings change when an item is assumed as unrated. The $I O I$ values that are individually excluded from the user vector are dynamically processed. The effect of each discarded rating was recorded as a residual over the dynamic mean or median. Thereafter, the static observation and dynamic approach were evaluated using the residual approach.

Figures 2 and 3 show the static and dynamic analyses based on the (A) mean and (B) median usage based on the ML100K and ML1M releases, respectively. The $\mathrm{x}$ - and $\mathrm{y}$-axis show the user ID and unique rating values, respectively. Each red dot statically indicates the mean or median values of all user ratings, whereas the blue dots show the deviation of the unit ratings from the static value. It may be observed in the median analysis that the blue dots were aggregated, while the outliers in the datasets were suppressed, indicating the superiority of the median over the mean in the presence of outliers.

2) Item independency analyses

Considering the dynamic approach regarding real-time systems, excluding the $I O I$ in the users' statistical calculations depends on the item's independence condition. Therefore, each particular item in the datasets was analyzed based on the independence. The leave-item-out approach emerges as a useful method because the items are independent of each other. Consequently, an item-based one-way analysis of variance (ANOVA) was performed. Each column (i.e., each item) was subjected to testing in the user $\times$ item matrix, validating their independencies. The ANOVA provides information about inter- and intra-group variations. By calculating the sum of squares (SS), degrees of freedom (df), and mean squared errors (MS), the F-test (the ratio of inter- and intra-group variability) is applied. Considering Tables 3 and 4, an analysis of the ML100K and ML1M releases is presented. The validity of item independence is proven by both the $\mathrm{F}>>1$ and probability $(\mathrm{P})$ values, which are obtained from the F-distribution. The lower the Pvalues, the higher the chances of strong evidence against the null hypothesis. $\mathrm{P}$-values $<<0.05$ (significance level) were obtained, indicating that the null hypothesis is rejected.

\section{B. Similarity and Prediction Equations}

The four touchstone similarity equations and prediction formula are considered in this section. Before the technical statements, an overview of the application perspective of the touchstone equations is provided.

PCC is within the scope of several studies. Music RS is among the most common applications [28]. In particular, considering music genre recommendations, $P C C$ has attracted attention. Additionally, there are other applications. Mukaka explains the management of medical data based on the utilization of PCC [29], as in other studies [30], [31]. Apart from these, book recommendations via PCC [32], [33], e-commerce applications [34], and academic paper RS [35] are intriguing alternatives. Other $P C C$ examples can be found in [36]-[39]. The most common application is movie-based RS [40]-[42], which is the motivation of the present work. Movie 
195

196

197

198

199

200

201

202

203

204

205

206

207

208

209

210

211

212

213

214

215

216

217

218

219

220

221

222

223

224

225

226

227

228

229

230

231

232

233

234

genre correlations were calculated using PCC by Kim et al. [43]. Hwang et al. also presented the details of the $P C C$, considering movie genre classification [44]. Nonetheless, $P C C$ has some disadvantages, considering the linear averaging procedures. Tan et al. indicated the underlying limitations of the PCC in reinforcing the effect of correlation. Subsequently, they proposed the resonance similarity between users by parametrizing the median of the rating values. They constructed a physical analogy between user similarities regarding simple harmonic motion in a coordinate system [45]. The mean of the rating vectors can be vulnerable to outliers and biases, as Garcin et al. emphasized the superiority of median-based rating aggregations over mean- and mode-based aggregations [46]. Therefore, the use of $M R C$ was proposed to suppress outliers in user ratings in the context of RS. COS is another frequently used method in RS owing to its simple calculation, and is encountered in movie-related applications [47], [48], research paper recommendation [49]-[51], cognitive similarity-based design [21], article suggestion system [52], and music RS [53]. Furthermore, JAC has been evaluated in several studies [19], [26], [54], [55]. JAC has an essential feature regarding binary rating analysis [56] and is considered a measure that does not treat absolute ratings [57]. From the perspective of merits and demerits of the relevant touchtone equations, the following detailed information is introduced. In general, PCC provides a concept of the presence, absence, and degree of correlation. It also provides feedback on positive and negative correlations. However, computationally PCC is complex owing to the complex algebraic requirements in its formula. It is also ineffective against outlier values. One merit of $M R C$ emerges with its median usage, whereas $P C C$ is based on the assumption of only linear correlation and is not the appropriate option for homogeneous data. Although $M R C$ shows similar features to $P C C$, it is a more suitable option, especially for data with outliers. However, demerits of $P C C$ are also valid for $M R C$. Using angle information, $C O S$ easily calculates the correlation between data, which are even quite far in terms of Euclidean distance. However, $\operatorname{COS}$ does not provide a concept about magnitude. Saranya et al. emphasized that $C O S$ is ineffective in capturing similar users who rated quite few items. Conversely, JAC has the merits of binary set processing. The utilization of $J A C$ is simple because the equation requires only the set operations, especially for the binary ratings. However, if a system has vectors of categorical or multiple-valued data, then $J A C$ requires a preprocessing step for the binarization [98], [99].

Together with the proposed $n I O I$ and $S W$ modifications over similarity equations, different combinations are interpreted by inferring the underlying affinity. Subsequently, the $P C C$ equation, $M R C, C O S$, and $J A C$ similarities are stated technically. Owing to the various performance metrics presented in Subsection $\mathrm{D}$, adequate weight-metric combinations are to be determined.

\section{1) Pearson correlation}

Pearson correlation is an acclaimed measure adopted in many data-mining approaches that address the similarity of measurement data. Regarding the user-based CF, the PCC is a tool used to define in-between user similarity by considering the item ratings. Pearson weighs all 
235

236

237

238

239

240

241

242

243

244

245

246

247

248

249

250

251

252

253

254

255

256

257

258

259

260

261

262

263

264

265

connected neighbors and calculates the degree of a linear relationship between two users. Thus, a weight for each correlated neighbor is derived, achieving a linear relationship by processing the deviation from the mean values, $\bar{r}$ [58]. Considering Eq. (1), the similarity formula between two users, $a$ and $u$, is indicated.

$$
w_{a, u}^{P C C}=\frac{\sum_{i \in\left(\mathrm{I}_{a} \cap \mathrm{I}_{u}\right)}\left(\left(r_{a, i}-\bar{r}_{a}\right) \times\left(r_{u, i}-\bar{r}_{u}\right)\right)}{\sqrt{\sum_{i \in\left(\mathrm{I}_{a} \cap \mathrm{I}_{u}\right)}\left(r_{a, i}-\bar{r}_{a}\right)^{2}} \times \sqrt{\sum_{i \in\left(\mathrm{I}_{a} \cap \mathrm{I}_{u}\right)}\left(r_{u, i}-\bar{r}_{u}\right)^{2}}} .
$$

2) Median-based robust correlation

Median-based robust correlation is a method that replaces the linear mean procedures with the median operation [59]-[61]. The utilization of the averages may suffer from the skewness problem [62], [63]. In addition, outliers can affect mean values. The $M R C$, which has the median of rating values instead of the averages similar to those in $P C C$, represents the suppression of outliers in the ratings of each user. Considering Eq. (2), the $M R C$ formula is as follows.

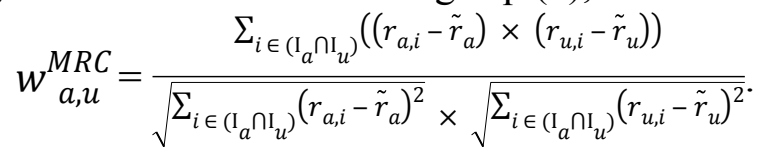

Contrary to the mean values of user ratings, $\bar{r}_{a}$ and $\bar{r}_{u}$ in Eq. (1), the median values, $\tilde{r}_{a}$ and $\tilde{r}_{u}$, represent the midpoints of the ratings. The formula is similar to $P C C$, and the median point of the user ratings is considered as a neutral mark.

3) Cosine similarity

Another similarity is based on the cosine function. By performing the Euclidean dot product, the cosine value between the two $n$-element vectors $\boldsymbol{A}$ and $\boldsymbol{B}$ can be determined. Thus, the similarity is based on $\boldsymbol{A} \cdot \boldsymbol{B}=\|\boldsymbol{A}\|\|\boldsymbol{B}\| \cos \Theta$. Considering the user-based similarity calculation via COS, the similarity weight between $a$ and $u$ can be measured as in Eq. (3).

$$
w_{a, u}^{C O S}=\frac{\boldsymbol{r}_{\boldsymbol{a}} \cdot \boldsymbol{r}_{\boldsymbol{u}}}{\left\|\boldsymbol{r}_{\boldsymbol{a}}\right\|\left\|\boldsymbol{r}_{\boldsymbol{u}}\right\|}
$$

Some other versions of the conventional COS have also been developed, such as the adjusted [64] and asymmetric cosine similarities [53].

\section{4) Jaccard similarity}

Jaccard similarity is a measure of common elements in two sets. The rating history of a user under test, $\boldsymbol{I}_{\boldsymbol{a}}$, and corresponding neighbor history, $\boldsymbol{I}_{\boldsymbol{u}}$, are calculated using Eq. (4). The JAC considers the two sets by having the ratio of their intersection to the union. The range of this similarity coefficient is $0 \leq w_{a, u} \leq 1$, where zero indicates that there are no common elements, whereas one implies that all the elements in the two sets are fully joint.

$$
w_{a, u}^{J A C}=\frac{\left|\boldsymbol{I}_{\boldsymbol{a}} \cap \boldsymbol{I}_{\boldsymbol{u}}\right|}{\left|\boldsymbol{I}_{\boldsymbol{a}} \cup \boldsymbol{I}_{\boldsymbol{u}}\right|}
$$

5) Prediction equation 
266

267

268

269

270

271

272

273

274

275

276

277

278

279

280

281

282

283

284

285

286

287

288

289

290

291

292

293

294

295

296

297

298

After the similarity calculations for all the best neighbor nominees, the obtained weights are sorted by denoting $w_{a, u^{*}}$. Thereafter, considering the sorted weights and $B N C$ limits, the best neighbors are determined. The prediction phase must be completed to achieve the recommendation score. The rating prediction formula, which is known as the mean centering approach [65]-[69], is given in Eq. (5).

$$
p_{a, i}=\bar{r}_{a}+\frac{\sum_{u^{*}=1}^{B N C}\left(\left(r_{u^{*}, i}-\bar{r}_{u^{*}}\right) \times w_{a, u}\right)}{\sum_{u^{*}=1}^{B N C} w_{a, u^{*}}} .
$$

\section{Modified Equations}

Considering the equations given in the previous section, we modified these formulas. As a result, the efficiency of RS was significantly improved under some circumstances.

The modifications were made based on two aims, including (i) to create a system model suitable for real-time applications and (ii) to boost the similarity weights. The former is related to the dynamicity, whereas the latter is related to the user-of-interest and its neighbor by considering their $C I C$ as a constant multiplier, thereby signified weights can be obtained.

Considering the first phenomenon, the already rated test item was discarded from the user-ofinterest rating history to predict the actual rating. Thus, during the mean or median calculations in formulas such as $P C C$ and $M R C$, the item is excluded as expected in real-time systems. This case is also valid for other measurements, such as $C O S$ and $J A C$, where the related item is removed from the vectors in progress. To indicate this phenomenon, we use the $n I O I$ subscript by denoting $\hat{a}$ in the equations. As explained in the first section, the negligence of the $n I O I$ in many other RS applications is thought to be due to runtime concerns in vast scientific tests. The second phenomenon is relative weight scaling, known as $S W$. This gives priority to a neighbor with more common ratings for the items. After calculating the co-rated item count, the weights in similarity calculations are signified using $C I C=\left|\mathrm{I}_{a} \cap \mathrm{I}_{u}\right|$, a constant multiplier [70]. There are other alternatives in the literature [11]-[16], [70]. For instance, Bellogín et al. [15] compared different user-user weighting schemes. That set in this study is known as user overlap, which calculates common item counts between user neighbors [71], considering Herlocker [72] and McLaughlin's significance weightings [73] together with trustworthiness [74] and trust deviation [75]. However, they either include extra parameters or require complex computations. Raeesi and Shajari compared the $S W$ strategies by underlining the user overlap, which demonstrated a higher efficiency, considering the error rates, although there were few arguments to process [71].

Regarding the modifications, each equation from the previous section is updated using the two abovementioned phenomena. Considering PCC, based on Eq. (1), Eq. (6) is obtained by 
299 excluding test-item bias. Subsequently, Eq. (7) is the signified version of Eq. (1) by applying 300 only $S W$.

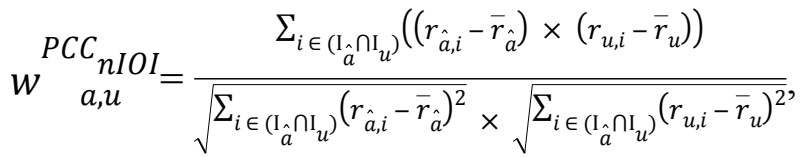

$$
\begin{aligned}
& w_{a, u}^{P C C^{S w}}=\left|\mathrm{I}_{a} \cap \mathrm{I}_{u}\right| \times w_{a, u}^{P C C} .
\end{aligned}
$$

301 The same approach is followed for the $M R C$ in the Eq. (8). The $S W$ multiplication is expressed in 302 Eq. (9).

$$
\begin{aligned}
& w_{a, u}^{M R C_{n I O I}}=\frac{\sum_{i \in\left(\mathrm{I}_{\hat{a}} \cap \mathrm{I}_{u} u\right.}\left(\left(r_{\hat{a}, i}-\tilde{r}_{\hat{a}}\right) \times\left(r_{u, i}-\tilde{r}_{u}\right)\right)}{\sqrt{\sum_{i \in\left(\mathrm{I}_{\hat{a}} \cap \mathrm{I}_{u}\right)}\left(r_{\hat{a}, i}-\tilde{r}_{\hat{a}}\right)^{2}} \times \sqrt{\sum_{i \in\left(\mathrm{I}_{\hat{a}} \cap \mathrm{I}_{u} u\right.}\left(r_{u, i}-\tilde{r}_{u}\right)^{2}}}, \\
& w_{a, u}^{M R C^{S W}}=\left|\mathrm{I}_{a} \cap \mathrm{I}_{u}\right| \times w_{a, u}^{M R C} .
\end{aligned}
$$

303 Regarding the COS, Eq. (10) shows the vector operations of the ratings in which the test-item 304 bias is discarded, and the $S W$ approach is described in Eq. (11).

$$
\begin{gathered}
{ }^{\operatorname{COS}_{n I O I}}=\frac{\boldsymbol{r}_{\hat{a}} \cdot \boldsymbol{r}_{\boldsymbol{u}}}{\left\|\boldsymbol{r}_{\hat{a}}\right\|\left\|\boldsymbol{r}_{\boldsymbol{u}}\right\|}, \\
w_{a, u}^{\operatorname{COS}_{a, u}^{S W}}=\left|\mathrm{I}_{a} \cap \mathrm{I}_{u}\right| \times w_{a, u}^{C O S} .
\end{gathered}
$$

305 Finally, JAC with the modifications is shown in Eq. (12) and (13).

$$
\begin{aligned}
& { }_{W}^{J A C_{n I O I}}=\frac{\left|\boldsymbol{I}_{\hat{\boldsymbol{a}}} \cap \boldsymbol{I}_{\boldsymbol{u}}\right|}{\left|\boldsymbol{I}_{\hat{\boldsymbol{a}}} \cup \boldsymbol{I}_{\boldsymbol{u}}\right|}, \\
& w_{a, u}^{J A C^{S w}}=\left|\mathrm{I}_{a} \cap \mathrm{I}_{u}\right| \times w_{a, u}^{J A C} .
\end{aligned}
$$

306 Although previous studies [11]-[15], [71]-[75] have presented a good understanding of $S W$

307

308

309

310

311 312 (14).

$$
w^{\text {SIMILARITY }} \underset{n I O I}{S W}=\left|\mathrm{I}_{\hat{a}} \bigcap \mathrm{I}_{u}\right| \times w^{\text {SIMILARITY }}{ }_{n I O I}
$$

313 The modified rating prediction formula is given by Eq. (15). Considering the $n I O I, \bar{r}_{a}$ is updated 314 compared with the original equation in Eq. (5).

$$
p_{a, i}=\bar{r}_{\hat{a}}+\frac{\sum_{u^{*}=1}^{B N C}\left(\left(r_{u^{*}, i}-\bar{r}_{u^{*}}\right) \times w_{a, u^{*}}\right)}{\sum_{u^{*}=1}^{B N C} w_{a, u^{*}}} .
$$


315

316

317

318

319

320

321

322

323

324

325

326

327

328

329

330

331

332

333

334

335

336

337

338

339

340

341

342

343

344

345

346

347

348

349

350

351

352

353

\section{Performance Metrics}

The final phase of the proposed RS design involves monitoring the running algorithm. Because the CF is an intersection of statistics and machine learning, conclusive information on its performance is necessary. Particularly, understanding the inter-relational achievement of similarity equations with implied modifications requires thorough performance monitoring through numerous metrics. Regarding this, we focus on two main groups: well-known metrics and preeminent metrics. The former includes the frequently practiced performance monitoring, whereas the latter is less known in the literature, but still prominent for RS.

\section{1) Well-known metrics}

The well-known metrics in Table 5 are applied to the framework to provide insight for further studies. The explanations of the listed metrics are briefly summarized as follows.

First, exact accuracy is a metric used to measure the exact matches of actual ratings $\left(r_{a, i}\right)$ and corresponding predictions $\left(p_{a, i}\right)$. The accuracy computation is considered for the predicted rating, controlling whether $p_{a, i}=r_{a, i}$ or $p_{a, i} \neq r_{a, i}$. Considering the frameworks that use $N$-scale ratings, exact accuracy can provide a precise observation. In addition, threshold accuracy has also been used following the binary decision of liked and disliked items. By denoting $r_{a, i} \in \mathbb{N}^{+}$or $r_{a, i} \in$ $\mathbb{R}^{+}$, where $\operatorname{argmax}\left(r_{a, i}\right)=N$ over $N$-scale ratings, $t \in \mathbb{R}^{+}$as a threshold value should be set satisfying $t<N$. Thereafter, the rating value $r$ compared to the threshold value $t$ is evaluated to label liked or disliked items in a binary sense [19].

Second, correctly predicted positive values are measured via sensitivity, which is also known as the recall or true positive rate. In addition, the measure of the actual positives is monitored by precision, namely the positive predictive value. Precision is referred to by Powers [76] as the true positive accuracy, indicating the confidence score. From sensitivity and precision, the F1measure is calculated using the harmonic mean. On the contrary, aside from the positive decisions made, negatives have also been considered. Thus, specificity (or inverse sensitivity) represents the proportion of real negative cases. Moreover, inverse precision (or negative predictive value), which is also known as the true negative value by Powers in [76], shows the predicted negative instances. The false discovery and omission rates were deduced complementarily from the maximum metric scores of the precision and inverse precision. Shani and Gunawardana assert that the false discovery rate can be an alternative control mechanism [77], which is the proportion of FP to the actual positives. Similarly, the false omission rate is the ratio of $F N$ to all negatives [78].

Sensitivity and specificity are attributed as the true positive and negative rates, respectively. Similarly, the fallout and miss rate represent the false positive and false negative rates, respectively. The irrelevant recommendation ratio is obtained via the fallout. The miss rate is the ratio of the items that are not recommended although they are relevant. A recent study performed fallout and miss rate by practicing a personalized nutrition recommendation study [79]. Similar 
354

355

356

357

358

359

360

361

362

363

364

365

366

367

368

369

370

371

372

373

374

375

376

377

378

379

380

381

382

383

384

385

386

387

388

389

390

391

392 to the F1-measure, the utilization of precision and recall by means of geometric mean also appears in the Fowlkes-Mallows index. Considering another recent study, Panda et al. discussed how to increase the Fowlkes-Mallows index similar to the F1-measure [80]. Balanced accuracy provides a better perspective for performance analyses, considering an imbalanced confusion matrix. Balanced accuracy is the arithmetic mean of sensitivity and specificity. To understand the algorithm efficiency, utilizing several metrics, such as balanced accuracy, results in considerable feedback.

The final metrics are the threat score and prevalence threshold. The former considers hits, misses, and false alarms in the confusion matrix [81]. The latter emphasizes a sharp change in the positive predictive value. The prevalence threshold with a more geometric interpretation of the performance measurement with a focus on positive and negative predictive values is provided in [82], and it has been applied to test analyses of Covid-19 screening [83].

The metrics constructed from the confusion matrix and the error metrics, such as mean absolute error (MAE), mean squared error (MSE), and root mean squared error (RMSE), are considered in this study. Li et al., in their privacy-preserving CF approach, measured performance using RMSE and MAE [20] as in Nguyen et al. [21]. The RMSE has demonstrated its efficiency in measuring error performance. For instance, considering the Netflix Prize competition, it was used as a vital indicator of the implementation [84].

2) Preeminent metrics

Although previous studies have given priority to F1-measure, precision, recall, and error-based measures [19]-[21], [85], some other performance metrics which are relatively less recognizable in RS also provide robust decisions. According to Chaaya et al., well-known metrics cause significant biases, and markedness, informedness, and Matthews correlation are noteworthy alternatives [86]. Because these preeminent metrics have limited utilization in the literature, they have been considered with a priority in this study. They consist of confusion matrix primitives as shown in Table 6. The definitions of these metrics are briefly reviewed below.

\section{i) Markedness}

The proportion of correct predictions is measured by markedness. This metric is free from an unbalanced confusion matrix. The markedness scores in the range $[-1,+1]$ and its associated formula is demonstrated in Eq. (16). Markedness can be a substitution of precision, which can be used as a tool that shows the status of the recommendation and "chance" [17]. To the best of our knowledge, markedness is one of the least considered metrics in the literature in the scope of RS science, although it supremely supplies information related to positive and negative predictive values. For instance, this phenomenon is known as DeltaP in the field of psychology, and Powers confirms that markedness is considered as a good predictor of human associative judgments [76], [87].

$$
\text { Markedness }=\text { Precision }+ \text { Inverse Precision }-1 .
$$


393 ii) Informedness

394 The second preeminent metric is informedness, which includes sensitivity and its inverse as 395 shown in Eq. (17). Informedness scores in the same range, [-1,+1], as in markedness [17]. This 396 metric is also known as the Youden's index because it differs from the accuracy, considering 397 imbalanced events in the confusion matrix. The returned score defines a perfect prediction by +1 , 398 or indicates the opposite by -1 [88]. The efforts of informedness in RS science are limited 399 although there are intriguing applications that practice this promising metric. Pilloni et al. 400 performed informedness in e-health recommendations [89]. Considering hotel recommendations, 401 informedness has also been used to check the performance of the multi-criteria system. Ebadi 402 and Krzyzak set two performance metrics as prediction- and decision-based, where informedness 403 was considered in the scope of decision-based metrics [90]. Regarding another research field, Marciano et al. utilized informedness in the context of genetic applications. This was considered as the relative level of confidence [91]. In addition, Layher et al. measured the performance of neuromorphic applications by assessing informedness [92].

$$
\text { Informedness }=\text { Sensitivity }+ \text { Inverse Sensitivity }-1 .
$$

407

408

iii) Matthews correlation

409

410 The Matthews correlation is a promising observation of binary labeling. Considering Eq. (18), a

411 wide implicit observation is obtained with a score in the range of $[-1,+1]$. The interpretation of

412 disagreement between the actual and predicted values. According to the score range, each 413 corresponding focus point is indicated by $+1,0$, and -1 , respectively [93]. Matthews Correlation Positive Predictive Value $\times$ True Positive Rate $\times$ - False Discovery Rate $\times$ False Negative Rate $\times$ $=\sqrt{\text { True Negative Rate } \times \text { Negative Predictive Value }}-\sqrt{\text { False Positive Rate } \times \text { False Omission Rate }}$

414 Matthews correlation is a combination of informedness and markedness. The former considers

415 how informed the classifier's decision with knowledge is compared to "chance" [76].

416

417

418

419

420

421

422

423

424

425

426

427

428 Alternatively, informedness is paraphrased as a probability with respect to a real variable rather than the "chance" [94]. Conversely, markedness carries information on how possibly the prediction variable will be marked by the true variable [92]. Overall, the Matthews correlation, as a geometric mean of informedness and markedness, shows the correlation between the prediction and true values. The occurrence of this metric is rare in the literature; nonetheless, some intriguing studies have been conducted, such as those on diet recommendations [95].

\section{Experimental Design}

This section describes how we processed the data for various similarity measurements. The overall algorithmic flow is introduced, including the modifications to the equations. The premising $B N C$ values were determined prior to its usage in the subsequent section. We first focus on the algorithm applied during the simulations. The algorithmic flow can guide any prospective RS scientist to follow the basic steps. The procedure for the proposed test 
429 package is summarized in Algorithm 1. The details related to several constant parameters, such 430 as test item count, cross-validation fold, neighbor counts, and liking threshold, are presented. 431 Running the algorithm for any user, five random items were considered. The $k$-fold cross432 validation technique was integrated with the implementation of repeated randomized test 433 attempts. In each independent analysis, the folds were shuffled, and the test items were 434 alternated. Regarding reliability, each test attempt was performed multiple times and averaged. 435 Utilizing the fine-tuned $\varepsilon$ parameter, an increased runtime may occur, especially in bulk tests. 436 Several previous studies have initiated this step size as $\varepsilon=50$ [96], $\varepsilon=10$ [23]-[25], or $\varepsilon=5$ 437 [11], [26]. In addition, Feng et al. measured different similarity measures by setting $\varepsilon=5$; 438 however, they focused only on the error metrics [18]. Bag et al. illustrated the performance of the 439 metrics using discrete $B N C$ values of 5, 20, 50, and 100 [19]. Considering our test package, the 440 fine-tuned neighbor step size $\varepsilon$, is set distinguishingly compared to the previous studies. $\varepsilon=1$ 441 was chosen to monitor the sensitivity of the tests, and the neighboring interval produced smooth 442 findings.

443 Furthermore, one of the main perspectives of previous efforts is to limit the computation time by 444 generalizing several parameters in the global scope of a development environment. Considering each iteration of the test package, although utilizing globally computed arguments reduces the runtime of experiments, it lacks the necessary dynamic perspective for real-time application imitation. Therefore, we performed Algorithm 1 for all similarity equations to examine this fallacy.

450 We applied the algorithm to two separate datasets to investigate the overall performance. A finetuned neighboring approach was performed for the best combination of the similarity equation and performance metrics. After selecting test items throughout steps one to three, all possible

452

453

454

455

456

457

458

459

460

461

462

463

464

465

466

467

468

similarity equations were called in step four. A clear picture of the equations and corresponding modifications for dynamicity and weight significance are presented in Table 7. A parametric neighborhood was applied from 1 to 100 users with a single increment as shown in step five. Regarding each loop iteration in step six, the best neighbors were selected based on the similarity score, which was sorted for all neighboring nominees, indicating the users who rated the test item. After the prediction calculation in step seven, the performance was evaluated in step eight. During the correlation computations, we emphasize the possible shortcomings of readily available functions on computing platforms. Correlation methods are mostly inline functions in a development environment. However, we strongly suggest checking the built-in functions for statistical parameter calculation, such as the mean and median. It is advised not to consider the statistics of only the co-rated items during the computations. It is more accurate to include the statistics of all items in analyzing general behavior and shared characteristics, especially in the context of dynamic RS [97].

\section{Results and Discussion}

In this section, several repeated randomized tests were analyzed considering various performance metrics. The preliminary findings related to the best-performing $B N C \mathrm{~s}$ are first given to provide

Peer) Comput. Sci. reviewing PDF | (CS-2021:05:61214:2:0:NEW 12 Oct 2021) 
469

470

471

472

473

474

475

476

477

478

479

480

481

482

483

484

485

486

487

488

489

490

491

492

493

494

495

496

497

498

499

500

501

502

503

504

505

506

507

508

guide to the subsequent sections. Then, the effect of sensitive neighboring interval selection for different similarity equations is performed, thereby measuring the importance of dynamicity and weight significance.

First, the best-performing $B N C$ values of the related performance monitoring are discovered considering each similarity measure in Table 7. After setting $B N C$ precisely, the observations under the various performance metrics are summarized in Tables 8 and 9. Table 8 shows the ML100K-based BNC values recorded for the dynamicity and weight significance approaches. Meanwhile, Table 9 presents the same analyses of the ML1M. These dataset-oriented analyses facilitate guidance for further metric comparisons in the subsequent section. Here, $B N C$ values inspected beforehand are utilized to interpret the adequate weight-metric combinations. Moreover, the preliminary results presented in Tables 8 and 9 highlight the effect of the $S W$ method in terms of $B N C$. For instance, $P C C$ benefits the reduced $B N C$ s with the best performance when $S W$ is applied. Excluding specificity and fallout in the ML100K, PCC has the advantage of the $S W$ method. As presented in Table 8, $P C C_{n I O I}^{s w}$ achieves the top performance when $B N C=17$ for markedness, Matthews correlation, F1-measure, threshold-based error metrics and accuracy, Fowlkes-Mallows index, threat score, inverse precision, sensitivity, miss rate, and false omission rate in the ML100K. Similarly, considering the ML1M, the same observation with $B N C=31$ is valid for markedness, Matthews correlation, F1-measure, threshold-based error metrics, exact accuracy, threshold accuracy, Fowlkes-Mallows index, threat score, inverse precision, sensitivity, miss rate, and false omission rate. Further, the same $B N C$ monitoring in terms of $M R C$ was performed. F1-measure, exact MAE, exact accuracy, Fowlkes-Mallows index, threat score, inverse precision, sensitivity, miss rate, and false omission rate benefit from $S W$ in terms of achieving lower BNCs in the ML100K. Half of the metrics showed their top performance for $B N C=27$ and 28. In contrast, relatively higher $B N C$ values are required in the ML1M for the top performances. More than half of the metrics work well for $B N C$ s 23 and 44 when the $S W$ approach is applied. The performance of COS in the ML100K in terms of the metrics that perform well with regard to lower $B N C \mathrm{~s}$ is similar to $M R C$. These are F1-measure, exact accuracy, Fowlkes-Mallows index, threat score, inverse precision, sensitivity, miss rate, and false omission rate. In the ML1M, COS is the least effective similarity equation in terms of the numbers of metrics, which benefit from $S W$ reducing the BNCs. However, JAC in the ML1M leads all other equations by having almost all metrics (except for informedness, exact $M S E$, and $R M S E$ ) performing well concerning lower $B N C$ s. Overall, the $S W$ approach is compatible with F1-measure, Fowlkes-Mallows index, threat score, inverse precision, sensitivity, miss rate, and false omission rate. This indicates lower BNCs when $S W$ is applied for all touchstone similarity equations both in the ML100K and ML1M. These observations can be visually inferred from Tables 8 and 9. In the following subsections, the evaluations related to the analyses of overall metrics, including the hybrid monitoring to achieve the adequate weightmetric combination, are discussed.

\section{A. Analyses of the Preeminent Metrics}

PeerJ Comput. Sci. reviewing PDF | (CS-2021:05:61214:2:0:NEW 12 Oct 2021) 
509 First, the preeminent metrics (such as informedness, markedness, and Matthews correlation) and

510 the F1-measure are utilized to show the comparative performance plots of all the similarity

511 equations in Table 7 using each individual metric. The ML100K and ML1M releases were

512 analyzed separately. Considering the plots, the $x$ - and $y$-axis represent the fine-tuned BNCs and

513 related metric output, respectively.

514 The statistical approach is depicted in this study to set a dynamic environment which requires a

515 more adaptive procedure. The results, based on hypothetical computations, can only determine

516 the maximum achievable top-performance of the dynamicity concept. We prove that dynamicity

517 deviates from the maximum reachable results. In the subsequent figures, the dashed lines

518 represent the theoretical perspective by including the global-only statistics, causing a fallacy.

519 Moreover, the solid lines represent dynamicity with the $n I O I$ approach. In addition, lines with

520 diamond marks illustrate the results free from the $S W$ approach, whereas the $S W$ adjustment can

521 be monitored through the unmarked lines.

522 Considering Figs. 4 and 5, performance plots of ML100K and ML1M are provided for the

523 preeminent metrics and F1-measure. Each row of subplots was compared to an equation-

524 dependent perspective. Analyzing the ML100K, the similarity equation with only the $S W$

525 modification achieves the best results for the PCC lines in black. Regarding the MRC (the lines

526 with a green color), the same dominance for the $S W$ methodology may be observed through all

527 the metrics. Although $S W$ does not boost the performance of the $C O S$ in all the metrics, plots

528 with $S W$ in $J A C$ show similar performance compared to those without $S W$.

529 Comparatively, we present the same metric performance throughout the similarity measures in

530 the context of ML1M as shown in Fig. 5. Including only the dynamic COS, all other similarities

531 with $S W$ increase the F1-measure performance. However, the performance in informedness

532 diminishes compared to that in Fig. 4. The top- and least-performing lines in each similarity

533 measure remain the same for markedness, Matthews correlation, and F1-measure as in the

534 ML100K. Nonetheless, regarding informedness, the effect of the $S W$ in the PCC and MRC

535 interchanges the least-performing similarity equation. Furthermore, dynamicity resulted in the

536 same expected outcomes in the ML1M analysis.

537

538

539

\section{B. Hybrid Monitoring Considering the Preeminent Metrics}

540

This subsection depicts the overall comparison of the compelled dynamicity with the applied

541 weight significance. Considering Fig. 6 (A), for ML100K, PCC is notably in the leading position

542 compared to other similarity measurements. The ranking for the rest of the similarity measures is difficult to generalize because the lines interchangeably depend on the $B N C$. Regarding

543 markedness, $M R C$ starts with better performance for fewer $B N C \mathrm{~s}$; however, the trend reverses

544 for $B N C>27$. In addition, measurements other than $M R C$ performed better for $B N C>40$. A

545 similar behavior is valid for the informedness and Matthews correlation; nevertheless, each of

546 them has a relatively greater $B N C$ threshold. Approximately, $B N C>75$ for informedness and

$547 B N C>60$ for Matthews correlation decayed the performance of the MRC. Considering the F1-

548 measure, a relatively stable performance was obtained for the measurements when $B N C>10$. 
549 Ranking the performance of the equations, the Fl-measure can be considered as the most stable

550

551

552

553

554

555

556

557

558

559

560

561

562

563

564

565

566

567

568

569

570

571

572

573

574

575

576

577

578

579

580

581

582

583

584

585

586

587

metric independent of the $B N C$ for the ML100K. This makes it appropriate comparisons of similarity measure performances without further $B N C$ considerations.

Regarding Fig. $6(B)$, the same hybrid monitoring of $n I O I$ and $S W$ is presented for the ML1M. The top-performing lines of the abovementioned preeminent metrics were still obtained via the PCC. The COS, compared to the others, has a relatively poor performance for the informedness metric. The performance ranking of the similarity equations remains more stable as a function of the $B N C$ compared to the ML100K. There are slight interchanges between $M R C$ and JAC only in informedness. Nonetheless, considering the others, the relative performance is independent of the $B N C$. Contrary to the significant interchanges in the ML100K, performance metrics maintain their relative positions in the ML1M. This stability finding can be a general interpretation, and it can be concluded that the larger the dataset is, the more stable the performance.

\section{Extensive Analysis of Other Metrics for Hybrid Monitoring}

The subsequent figures illustrate the extensive analysis of other metrics frequently evaluated in the literature. First, the ML100K plots with hybrid monitoring are presented in Fig. 7. Regarding accuracy-based metrics, both the exact (sensitive rating prediction) and binary (liked or disliked labeling) performances were monitored. The PCC had a relatively higher accuracy margin of approximately 0.05 for both metrics. Another accuracy calculation is performed extensively in this study. Considering balanced accuracy, the PCC outperformed for all the BNC $\mathrm{s}$, whereas the $M R C$ diminished. Similarly, the error metrics were measured, considering both exact and binary performances. As expected, the binary prediction error rates were lower than the exact prediction error rates. Both are plotted using MAE, MSE, and RMSE. Considering the binary prediction error, the PCC achieved the lowest possible error rates. Nonetheless, regarding the exact accuracy metric, the top performance for low error rates was interchangeable based on the neighborhood. Approximately $B N C>40, B N C>20$, and $B N C>20$ for MSE, MAE, and $R M S E$, respectively, yielded lower error rates with the $J A C$ measure.

In addition, the Fowlkes-Mallows index shows that $P C C$ and $C O S$ achieve outstanding performances, whereas in general, the $M R C$ has poor performance. Considering the threat score, the hits of user dislikes were not included; however, the ratio of liking matches concerning the misses is checked. Similarity measurement rankings are relatively stable after $B N C=10$ and $P C C$ was an adequate measure, while $M R C$ fell behind. This implies that, after the $B N C$ value of 10 , the ranking of the metric values from similarity equations relative to the $y$-axis remains stable.

This study also discusses different performance checks based on the interdisciplinary applications discussed previously. Considering the context of RS, we propose the application of a prevalence threshold that sources compound information, including several associated metrics inferred as $(\sqrt{\text { sensitivity } \times \text { fallout }}-$ fallout $) /$ Informedness. Similar to the error metrics, the lesser the prevalence threshold value is, the more exactitude accomplished. Although higher

Peer] Comput. Sci. reviewing PDF | (CS-2021:05:61214:2:0:NEW 12 Oct 2021) 
588 values are obtained in the COS compared to the others, the $P C C$ has proven its superiority with 589 lower rates.

590 Some metrics, such as sensitivity and specificity, provide information on how likely the top- $n$ 591 items match the user's taste or vice versa. Correctly identified positives (i.e., sensitivity) perform 592 well for the lower $B N C \mathrm{~s}$, and the $C O S$ measure leads with a peak of approximately $B N C=9$. 593 Correctly identified negatives (i.e., specificity) are distinctively better as the neighbor count 594 increases for $C O S$ and $J A C$, whereas $P C C$ and $M R C$ are relatively stable.

595 The last two rows of the subplots are complementary metric couples. The metrics of sensitivity 596 and miss rate; specificity and fallout; precision and false discovery rate; inverse precision and 597 false omission rate complete each other. Considering specificity, precision, and inverse 598 precision, $P C C$ performs adequately, which can be verified from fallout, false discovery rate, and 599 false omission rate.

600 The ML1M evaluation is presented comparatively to the previous findings of the ML100K. 601 Regarding Fig. 8, the analyses are illustrated for the same evaluated metrics. The trend in the 602 exact accuracy was similar to that of the ML100K with greater scores. The PCC puts a margin, 603

604 whereas the others perform closer to each other with slightly lower values compared to the $P C C$.

605

606

607

608

609

610

611 In addition, the exact prediction error metrics generally result in a reduced numerical range. The most erroneous metric is the COS, which is valid for both exact and binary prediction error metrics. The error performance in the ML1M is relatively stable, and the PCC is still a less fragile metric for binary prediction. Considering binary analyses, similarity measures are homogenously ranked again with the dominance of the PCC.

Furthermore, the Fowlkes-Mallows index shows an increased range of scoring with respect to the ML100K findings. Regarding the threat score, it was observed that the $M R C$ significantly

612 improved compared to the others. Considering the prevalence threshold, COS had a higher margin than the others compared to the performances in the previous analysis. Moreover, although the $C O S$ has a good sensitivity observation, it performs worse in terms of specificity and precision, as demonstrated in the previous findings. This indicates that the $\operatorname{COS}$ suffers from $a$

616 The monitoring of smooth sensitivity in the ML1M is an important feedback because it is a 617 component of some compound metrics. On the contrary, an indicative finding from the 618 comparison of both releases is the behavior of the specificity and fallout metric couples. 619 Considering the ML100K, a relatively more stable distribution is monitored for increasing the $620 B N C$ values, whereas in the ML1M, the behavior becomes unstable. Lastly, whereas JAC for 621 precision increases in the ranking compared to the previous findings, the opposite is valid for the 622 inverse precision.

623

\section{Adequate Weight-Metric Combination of the Top-Performing BNCs}

625 Having represented all the plots, we summarize the test results using a tabular structure for a 626 compact heat-map presentation. Considering Tables 10 and 11, the performance metrics for each 
627 similarity measurement are visualized in a colored format ${ }^{2}$. Considering the preliminarily

628 explanations in the third section, the selected $B N C$ s achieving the top performances are added to

629 the tables, highlighting the main motivation of our study: the decision of adequate weight-metric

630 combinations. Each metric is processed through column-wise coloring to make the comparison

631 easier. Therefore, each coloring is evaluated within its own column. This indicates that the same

632 color may correspond to different values in other columns; nevertheless, only a single column

633 should be considered to interpret the coloring for any metric. The comparison of the similarity

634 methods in the vertical direction is targeted, considering the neighborhoods. At the end of each

635 heat-map table, the minimum and maximum values referenced in the coloring of the relevant

636 column are shown. The tables demonstrate the comparison by addressing the different

637 correlation equations over the outstanding neighborhoods; thereby, comparing approaches such

638 as dynamicity and $S W$, considering each independent metric. The cells shaded in green indicate

639 the effectiveness of the appropriate combination. We present the results using both the $S W$ -

640 induced dynamic equations and plain dynamicity; hence, the effect of weight boosting is

641 monitored.

642 All the other test outcomes are found in our code repository ${ }^{3}$. We have prepared a fully detailed

643 supplementary material to include all the outcomes. Any RS researcher can benefit from the

644 prepared document for such purposes (e.g., the selection of $B N C \mathrm{~s}$, enhanced similarity measure

645 conditions, etc.), and every iteration in the test package has been logged into the abovementioned

646 document.

647 The colorized tables are organized by grouping the column names attributed to the performance

648 metrics. The first group is the prioritized preeminent metrics in this study. Subsequently, the

649 error-based metrics are combined. The third group is accuracy-based metrics, and the final

650 group is the rest of the metrics, which are frequently used in the literature, including

651 interdisciplinary applications. This method of representation determines the consistency of each

652 similarity equation, considering the groupings. Furthermore, because the tables are multi-

653 dimensional, they include metrics, correlation methods, and multiple parameters, such as $B N C$,

654 dynamicity, and $S W$. Because the neighborhood calculation makes the tests dependent on a

655 parameter in recommendation systems, the performance of the correlation is better if it is less

656 dependent on the neighboring users. Therefore, column-wise homogeneity indicates less

657 dependence on the $B N C$ range. Remarkably, the homogeneous column-wise scoring highlights

658 the $B N C$-free performance of any similarity equation. For instance, the $J A C$ equation with $S W$

659 generally maintained its stability in each metric group, considering smooth coloring.

660 Remarkably, homogeneous scoring highlights the overall performance of any similarity equation.

661 In a general view, if an RS design targets only the recommendations of preferable items, the COS

662 may be a suitable similarity measure. Metrics that do not address TN values such as F1-measure,

2 The fractional values in the table are displayed based on three significant digits. The heat-map coloring is achieved according to full precision.

${ }^{3}$ The supplementary material containing the complete results of the whole test package can be accessed from the repository given in the Acknowledgments. 
663 Fowlkes-Mallows index, threat score, sensitivity, and miss rate feedback the indicative scores

664 when combined with the COS. Conversely, the COS with the $S W$ approach, exhibited a

665 homogeneity of the heat-map tones, which deteriorates while transitioning between two

666 correlation methods. Although some metrics have the advantage of $S W$, the $C O S$ is not

667 completely compatible with the $S W$ approach. Conversely, the beneficial impact of the $S W$ can

668 be observed through all the other equations. Overall, the PCC is the most appealing metric.

669 Although the PCC considers linear correlation, it suitably fits into the five-star rating analyses.

$670 P C C$ with $S W$ can be generalized as one of the adequate similarity equations, whereas $M R C$

671 without $S W$ became the least performing equation as the harsh red background color indicates.

672 The $M R C$ without $S W$ showed the most inadequate performance as shown in Table 10; therefore,

673 the weighting method for the $M R C$ utilization is highly recommended for the ML100K, whereas

674 the case is slightly different for the ML1M.

675

\section{Conclusions}

677 This paper presented an experimental perspective for interpreting the interrelations between

678 similarity equations and performance metrics. The most indicative highlight of this article is the

679 necessity of a dynamic approach by performing independent computations. The misleading

680 effect of test-item bias has been emphasized in our analyses. It has been unveiled how this pitfall

681 can demarcate the results. The test-item bias in the training phase results in hazardous outcomes,

682 and the upper limit a system can reach was determined. Another highlight is the impact of

683 similarity weighting. All combinations of the modifications were monitored experimentally. The

684 overall evaluation was inferred from multiple simulations. In addition, we have conducted a fine-

685 tuned neighborhood analysis on the weight-metric combinations. The limit of the $B N C$ can be

686 deduced from our graphical interpretations. Furthermore, our remarks have been profoundly

687 demonstrated in the heat-map tables with the best-performing neighborhood surveyed by using

688 intercrossing similarity equations and specific metrics. Overall, the clearly back-end of any RS

689 design can be developed using the same procedures applied throughout this study. Any dataset

690 can be adaptively examined via the open-source code (as indicated in the Acknowledgments

691 section) of our framework. Starting from the fine-tuned neighborhood, the test-item bias

692 mitigation approach can be thoroughly followed with and without the $S W$ method. We believe

693 that further studies in RS science can benefit from the findings of this study. Considering future

694 studies, any other metadata or features, such as user demographics and item details, can be

695 included to enhance our framework.

696

\section{Acknowledgments}

698 A reproducible code run can be directly executed on the Code Ocean platform. Moreover, both

699

700

701

702 the open-source code of the whole package and the supplementary material, supplementaryMaterial_allResults.xlsx, have been attached to the https://github.com/savasokyay/AdequateWeightMetricDynamicCF repository. The supplementary material contains detailed results, including all the neighbors from the top 
703 performing to the least performing metrics. This file can easily be adapted by filtering and

704 sorting the fields.

705

706

707

708

709

710

711

\section{References}

[1] P. Kazienko, K. Musiał, and T. Kajdanowicz, "Multidimensional social network in the social recommender system," IEEE Trans. Syst. Man, Cybern. Part A: Systems and Humans, vol. 41, no. 4, pp. 746-759, Jul. 2011.

712

[2] A. Calero Valdez and M. Ziefle, "The users' perspective on the privacy-utility trade-offs in health recommender systems," Int. J. Hum. Comput. Stud., vol. 121, pp. 108-121, Jan. 2019.

713 system for computer science publications," Knowledge-Based Syst., vol. 157, pp. 1-9, Oct. 2018.

714 [4] I. Andjelkovic, D. Parra, and J. O’Donovan, "Moodplay: Interactive music

715 recommendation based on artists' mood similarity," Int. J. Hum. Comput. Stud., vol. 121, pp.

716 142-159, Jan. 2019.

717 [5] Ò. Celma and P. Herrera, "A new approach to evaluating novel recommendations," in

718 RecSys'08 Proc. 2008 ACM Conf. Recomm. Syst., Lausanne, Switzerland, 2008, pp. 179-186.

719 [6] M. N. Moreno, S. Segrera, V. F. López, M. D. Muñoz, and A. L. Sánchez, "Movie 720 recommendation framework using associative classification and a domain ontology," in Hybrid

721 Artificial Intelligent Systems, $8^{\text {th }}$ International Conf., Salamanca, Spain, 2013, pp. 122-131.

722 [7] F. O. Isinkaye, Y. O. Folajimi, and B. A. Ojokoh, "Recommendation systems: Principles, 723 methods and evaluation," Egypt. Informatics J., vol. 16, no. 3, pp. 261-273, Nov. 2015.

724 [8] Y. Wang, M. Wang, and W. Xu, "A sentiment-enhanced hybrid recommender system for 725 movie recommendation: A big data analytics framework," Wirel. Commun. Mob. Comput., vol. 726 2018, pp. 1-9, Mar. 2018.

727 [9] Grouplens, "MovieLens," 1992, [Online]. Available:

728 https://grouplens.org/datasets/movielens/

729 [10] Netflix, "Netflix price dataset," 2009, [Online]. Available: https:/www.netflixprize.com/

730 [11] M. A. Ghazanfar and A. Prugel-Bennett, "Novel significance weighting schemes for 731 collaborative filtering: Generating improved recommendations in sparse environments," in 732 International Conference on Data Mining, DMIN 2010, Las Vegas, USA, 2010.

733 [12] C. A. Levinas, "An analysis of memory based collaborative filtering recommender 734 systems with improvement proposals," M.S. thesis, Universitat Politècnica de Catalunya, 2014. 735 [13] B. Zhang and B. Yuan, "Improved collaborative filtering recommendation algorithm of 736 similarity measure," in AIP Conf. Proc., Hangzhou, China, 2017.

737 [14] M. Gao, Y. Fu, Y. Chen, and F. Jiang, "User-weight model for item-based 738 recommendation systems," J. Softw., vol. 7, no. 9, pp. 2133-2140, Sept. 2012.

739 [15] A. Bellogín, P. Castells, I. Cantador, "Neighbor selection and weighting in user-based 740 collaborative filtering: A performance prediction approach," ACM Trans. on the Web, no. 1, 741 Mar. 2014. 
742 [16] L. Zhang, Q. Wei, L. Zhang, B. Wang, and W. H. Ho, "Diversity balancing for two-stage 743 collaborative filtering in recommender systems," Appl. Sci., vol. 10, no. 4, pp. 1-16, Feb. 2020.

744 [17] G. Schröder, M. Thiele, and W. Lehner, "Setting goals and choosing metrics for 745 recommender system evaluations," in UCERSTI2 workshop at the $5^{\text {th }}$ ACM conference on 746 recommender systems, Chicago, USA, 2011.

747 [18] J. Feng, X. Fengs, N. Zhang, and J. Peng, “An improved collaborative filtering method 748 based on similarity," PLoS One, vol. 13, no. 9, pp. 1-18, Sept. 2018.

749 [19] S. Bag, S. K. Kumar, and M. K. Tiwari, "An efficient recommendation generation using 750 relevant Jaccard similarity,” Inf. Sci., vol. 483, pp. 53-64, Jan. 2019.

751 [20] D. Li, C. Chen, Q. Lv, L. Shang, Y. Zhao, T. Lu, and N. Gu, "An algorithm for efficient 752 privacy-preserving item-based collaborative filtering," Futur. Gener. Comput. Syst., vol. 55, pp. 753 311-320, Dec. 2014.

754 [21] L. V. Nguyen, M. S. Hong, J. J. Jung, and B. S. Sohn, “Cognitive similarity-based 755 collaborative filtering recommendation system," Appl. Sci., vol. 10, no. 12, pp. 1-14, Jun. 2020.

756 [22] T. Arsan, E. Koksal, and Z. Bozkus, "Comparison of collaborative filtering algorithms 757 with various similarity measures for movie recommendation," Int. J. Comput. Sci. Eng. Appl., 758 vol. 6, no. 3, pp. 1-20, Jun. 2016.

759 [23] J. L. Sánchez, F. Serradilla, E. Martínez, and J. Bobadilla, "Choice of metrics used in 760 collaborative filtering and their impact on recommender systems," in $2^{\text {nd }}$ IEEE Int. Conf. Digit. 761 Ecosyst. Technol., Phitsanuloke, Thailand, 2008, pp. 432-436.

762 [24] H. Liu, Z. Hu, A. Mian, H. Tian, and X. Zhu, "A new user similarity model to improve 763 the accuracy of collaborative filtering," Knowledge-Based Syst., vol. 56, pp. 156-166, Nov.

7642013.

765 [25] B. H. Huang and B. R. Dai, "A weighted distance similarity model to improve the 766 accuracy of collaborative recommender system," in IEEE Int. Conf. on Mob. Data Manag., 767 Pittsburgh, PA, USA, 2015, pp. 104-109.

768 [26] S. B. Sun, Z. H. Zhang, X. L. Dong, H. R. Zhang, T. J. Li, L. Zhang, and F. Min, 769 "Integrating triangle and Jaccard similarities for recommendation," PLoS One, vol. 12, no. 8, pp. 770 1-16, Aug. 2017.

771 [27] F. M. Harper and J. A. Konstan, "The movielens datasets: History and context," ACM 772 Trans. Interact. Intell. Syst., vol. 5, no. 4, pp. 1-19, Dec. 2015.

773 [28] U. Kuzelewska and R. Ducki, "Collaborative filtering recommender systems in music 774 recommendation,” Adv. in Comp. Sci. Res., vol. 10, pp. 67-79, 2013.

775 [29] M. M. Mukaka, "Statistics corner: A guide to appropriate use of correlation coefficient in 776 medical research," Malawi Med. J., vol. 24, no. 3, pp. 69-71, Sept. 2012.

777 [30] H. Akoglu, "User's guide to correlation coefficients," Turkish J. Emerg. Med., vol. 18, 778 no. 3, pp. 91-93, Sept. 2018.

779 [31] H. A. Miot, "Correlation analysis in clinical and experimental studies," J. Vasc. Bras., 780 vol. 17, no. 4, pp. 275-279, 2018. 
781 [32] N. Kurmashov, K. Latuta, and A. Nussipbekov, "Online book recommendation system," 782 in Int. Conf. Electron. Comput. Computa. ICECCO, Almaty, Kazakhstan, 2016.

783 [33] N. Sivaramakrishnan, V. Subramaniyaswamy, S. Arunkumar, A. Renugadevi, and K. K. 784 Ashikamai, "Neighborhood-based approach of collaborative filtering techniques for book 785 recommendation system," Int. J. Pure Appl. Math., vol. 119, no. 12, pp. 13241-13250, 2018.

786 [34] T. Q. Lee, Y. Park, and Y. T. Park, "A time-based approach to effective recommender 787 systems using implicit feedback,” Expert Syst. Appl., vol. 34, no. 4, pp. 3055-3062, May 2008.

788 [35] J. Lee, K. Lee, and J. G. Kim, "Personalized academic research paper recommendation 789 system,” 2013, Accessed on: Oct., 2020, [Online]. Available: http://arXiv:1304.5457

790 [36] Adiyansjah, A. A. S. Gunawan, and D. Suhartono, "Music recommender system based on 791 genre using convolutional recurrent neural networks," in $4^{\text {th }}$ Int. Conf. on Comp. Sci. Comput.

792 Intelli. (ICCSCI), vol. 157, 2019, pp. 99-109.

793 [37] Z. Cataltepe and B. Altinel, "Music recommendation by modeling user's preferred 794 perspectives of content, singer/genre and popularity," in Collaborative and Social Information

795 Retrieval and Access: Techniques for Improved User Modeling, edited by Max Chevalier, et al., 796 IGI Global, 2009, pp. 203-221.

797 [38] N. Sigg, "An investigation into the relationship between music preference, personality 798 and psychological wellbeing," M.S. thesis, Auckland University of Technology, 2009.

799 [39] D. Shepherd and N. Sigg, "Music preference, social identity, and self-esteem," Music 800 Perception, vol. 32, no. 5, pp. 507-514, 2015.

801 [40] S. Dhawan, K. Singh, and Jyoti, "High rating recent preferences based recommendation 802 system," in $4^{\text {th }}$ Int. Conf. on Eco. Comp. and Comm. Sys. (ICECCS), vol. 70, 2015, pp. 259-264.

803 [41] K. Madadipouya, "A location-based movie recommender system using collaborative 804 filtering," Int. J. Found. Comput. Sci. Technol., vol. 5, no. 4, pp. 13-19, Jul. 2015.

805 [42] L. Sheugh and S. H. Alizadeh, "A note on Pearson correlation coefficient as a metric of 806 similarity in recommender system," in AI \& Robot. (IRANOPEN), Qazvin, Iran, 2015.

807 [43] K. R. Kim, J. H. Lee, J. H. Byeon, and N. M. Moon, "Recommender system using the 808 movie genre similarity in mobile service," in $4^{\text {th }}$ Int. Conf. Multimed. Ubiquitous Eng., Cebu, 809 Philippines, 2010.

810 [44] T. G. Hwang, C. S. Park, J. H. Hong, and S. K. Kim, “An algorithm for movie 811 classification and recommendation using genre correlation," Multimed. Tools Appl., vol. 75, pp.

812 12843-12858, Apr. 2016.

813 [45] Z. Tan and L. He, "An efficient similarity measure for user-based collaborative filtering 814 recommender systems inspired by the physical resonance principle," IEEE Access, vol. 5, pp. 815 27211-27228, 2017.

816 [46] F. Garcin, B. Faltings, R. Jurca, and N. Joswig, "Rating aggregation in collaborative

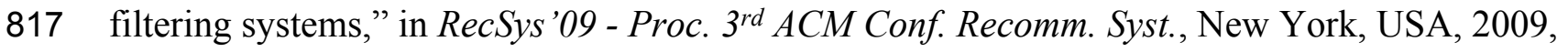
818 pp. 349-352. 
819 [47] R. H. Singh, S. Maurya, T. Tripathi, T. Narula, and G. Srivastav, "Movie 820 recommendation system using cosine similarity and KNN," Int. J. Eng. Adv. Technol., vol. 9, no. 821 5, pp. 556-559, Jun. 2020.

822 [48] I. S. Wahyudi, A. Affandi, and M. Hariadi, "Recommender engine using cosine similarity 823 based on alternating least square-weight regularization," in 15th Int. Conf. Qual. Res. Int. Symp.

824 Electr. Comput. Eng., Nusa Dua, Bali, Indonesia, 2017, pp. 256-261.

825 [49] S. Philip, P. B. Shola, and A. O. John, "Application of content-based approach in 826 research paper recommendation system for a digital library," Int. J. Adv. Comput. Sci. Appl., vol. 827 5, no. 10, pp. 37-40, 2014.

828 [50] S. Ahmad and M. T. Afzal, "Combining metadata and co-citations for recommending 829 related papers," Turkish J. Electr. Eng. Comput. Sci., vol. 28, pp. 1519-1534, 2020.

830 [51] A. Samad, M. A. Islam, M. A. Iqbal, and M. Aleem, "Centrality-based paper citation 831 recommender system," EAI Endorsed Trans. on Ind. Netw. and Intell. Sys., vol. 6, no. 19, pp. 1832 10, Jun. 2019.

833 [52] R. LVN, Q. Wang, and J. D. Raj, "Recommending news articles using cosine similarity 834 function," in Proc. SAS Ins. Global Forum, 2014, pp. 1-7.

835 [53] F. Aiolli, "Efficient top-N recommendation for very large scale binary rated datasets," in 836 RecSys'13 - Proc. 7th ACM Conf. Recomm. Syst., Hong Kong, China, 2013, pp. 273-280.

837 [54] L. Meilian, Q. Zhen, C. Yiming, L. Zhichao, and W. M. State, "Scalable news 838 recommendation using multi-dimensional similarity and Jaccard-Kmeans clustering," J. Syst.

839 Softw., vol. 95, pp. 242-251, May 2014.

840 [55] A. Rana and K. Deeba, "Online book recommendation system using collaborative 841 filtering (with Jaccard similarity),” J. Phys. Conf. Ser., vol. 1362, pp. 1-8, 2019.

842 [56] L. Zahrotun, "Comparison Jaccard similarity, cosine similarity and combined both of the 843 data clustering with shared nearest neighbor method," Comput. Eng. Appl. J., vol. 5, no. 1, pp.

844 11-18, Feb. 2016.

845 [57] N. F. AL-Bakri and S. H. Hashim, "A study on the accuracy of prediction in

846 recommendation system based on similarity measures," Baghdad Sci. J., vol. 16, no. 1, pp. 263847 269, Mar. 2019.

848 [58] K. Pearson, "Contributions to the mathematical theory of evolution," Philosophical 849 Trans. of the Royal Soc. of London, vol. (A.)185, pp. 71-110, Nov. 1893.

850 [59] G. L. Shevlyakov, "On robust estimation of a correlation coefficient," J. Math. Sci., vol. 85183 , no. 3, pp. 434-438, 1997.

852 [60] G. Shevlyakov and P. Smirnov, "Robust estimation of the correlation coefficient: An 853 attempt of survey," Austrian J. of Stat., vol. 40, no. 1\&2, pp. 147-156, 2011.

854 [61] V. R. Pasman and G. L. Shevlyakov, "Robust methods of estimating the correlation 855 coefficient," Avtomat. i Telemekh., Issue 3, pp. 70-80, 1987.

856 [62] K. Pearson, "Contributions to the mathematical theory of evolution-II. Skew variation in 857 homogeneous material," Philosophical Trans. of the Royal Soc. of London, vol. (A.)186, pp. 858 343-414, Jan. 1895. 
859 [63] M. Sato, "Some remarks on the mean, median, mode and skewness," Austrian J. of Stat., 860 vol. 39, no. 2, pp. 219-224, Jan. 1997.

861 [64] M. Gao, Z. Wu, and F. Jiang, "Userrank for item-based collaborative filtering 862 recommendation,” Inf. Process. Lett., vol. 111, no. 9, pp. 440-446, Apr. 2011.

863 [65] A. Saric, M. Hadzikadic, and D. Wilson, "Alternative formulas for rating prediction using 864 collaborative filtering," in Int. Symp. on Meth. for Intell. Sys. (ISMIS), 2009, pp. 301-310.

865 [66] H. Zeybek and C. Kaleli, "Dynamic k neighbor selection for collaborative filtering," 866 Anadolu Univ. J. Sci. Technol. A - Appl. Sci. Eng., vol. 19, no. 2, pp. 303-315, 2018.

867 [67] B. Sarwar, G. Karypis, J. Konstan, and J. Riedl, "Item-based collaborative filtering 868 recommendation algorithms," in Proc. of the 10th Int. Conf. on WWW, Hong Kong, 2001, pp. 869 285-295.

870 [68] J. Wu, L. Chen, Y. Feng, Z. Zheng, M. C. Zhou, and Z. Wu, "Predicting quality of 871 service for selection by neighborhood-based collaborative filtering," IEEE Trans. Syst. Man, 872 Cybern.: Sys., vol. 43, no. 2, pp. 428-439, Mar. 2013.

873 [69] P. K. Singh, M. Sinha, S. Das, and P. Choudhury, "Enhancing recommendation accuracy 874 of item-based collaborative filtering using Bhattacharyya coefficient and most similar item," 875 Appl. Intell., vol. 50, pp. 4708-4731, Aug. 2020.

876 [70] S. Okyay and S. Aygün, "A study of static and dynamic significance weighting 877 multipliers on the Pearson correlation for collaborative filtering," European J. of Sci. and Tech., 878 vol. Spec. Iss., pp. 270-275, 2020.

879 [71] M. Raeesi and M. Shajari, "An enhanced significance weighting approach for 880 collaborative filtering," in $6^{\text {th }}$ Int. Symp. Telecommun. (IST'12), Tehran, Iran, 2012, pp. 1165-

8811169.

882 [72] J. Herlocker, J. A. Konstan, and J. Riedl, "An empirical analysis of design choices in 883 neighborhood-based collaborative filtering algorithms," Inf. Retr., vol. 5, no. 4, pp. 287-310, 884 Jan. 2002.

885 [73] M. R. McLaughlin and J. L. Herlocker, "A collaborative filtering algorithm and 886 evaluation metric that accurately model the user experience," in Proc. of the 27th Annual Int. 887 ACM SIGIR Conf. on Res. and Dev. in Info. Retr., Sheffield, United Kingdom, 2004, pp. 329888336.

889 [74] J. Weng, C. Miao, and A. Goh, "Improving collaborative filtering with trust-based 890 metrics," in Proc. of ACM Symp on Appl. Comp., Dijon, France, 2006, pp. 1860-1864.

891 [75] C. S. Hwang and Y. P. Chen, "Using trust in collaborative filtering recommendation," in 892 Int. Conf. on Ind., Eng. and other App.s of Appl. Intell Sys IEA/AIE, Kyoto, Japan, 2007, pp. 893 1052-1060.

894 [76] D. M. W. Powers, "Evaluation: From precision, recall and F-factor to ROC, 895 informedness, markedness \& correlation," Technical Report SIE-07-001, School of Info. and 896 Eng., Flinders Uni., Adelaide, South Australia, pp. 1-24, Dec. 2007.

897 [77] G. Shani and A. Gunawardana, "Evaluating recommendation systems," Recomm. Syst. 898 Handb., pp. 257-297, 2011. 
899 [78] M. Mukhtar, S. S. Ali, S. A. Boshara, A. Albertini, S. Monnerat, P. Bessell, Y. Mori, Y. 900 Kubota., J. M. Ndung'u, and I. Cruz, "Sensitive and less invasive confirmatory diagnosis of 901 visceral leishmaniasis in Sudan using loop-mediated isothermal amplification (LAMP)," PLoS 902 Negl. Trop. Dis., vol. 12, no. 2, pp. 1-14, Feb. 2018.

903 [79] K. R. Devi, J. Bhavithra, and A. Saradha, "Personalized nutrition recommendation for 904 diabetic patients using improved K-means and Krill-Herd optimization," Int. J. Sci. Tech. Res., 905 vol. 9, no. 3, pp. 1076-1083, Mar. 2020.

906 [80] S. K. Panda, S. K. Bhoi, and M. Singh, "A collaborative filtering recommendation 907 algorithm based on normalization approach," J. Ambient Intell. Humaniz. Comput., vol. 11, no. 908 11, pp. 4643-4665, Jan. 2020.

909 [81] R. J. Hogan, C. A. T. Ferro, I. T. Jolliffe, and D. B. Stephenson, "Equitability revisited: 910 Why the 'equitable threat score' is not equitable," Weather and Forecasting, vol. 25, no. 2, pp. 911 710-726, Apr. 2010.

912 [82] J. Balayla, "Prevalence threshold $\left(\varphi_{\mathrm{e}}\right)$ and the geometry of screening curves," PLoS One, 913 vol. 15, no. 10, pp. 1-12, Oct. 2020.

914 [83] J. Balayla, A. Lasry, Y. Gil, and A. V. Perel, "Prevalence threshold and temporal 915 interpretation of screening tests: The example of the SARS-CoV-2 (COVID-19) pandemic," 916 2020, Accessed on: Oct., 2020, [Online]. Available: https://doi.org/10.1101/2020.05.17.

91720104927

918 [84] R. M. Bell and Y. Koren, "Lessons from the Netflix prize challenge," ACM SIGKDD 919 Explor. Newsl., vol. 9, no. 2, pp. 75-79, Dec. 2007.

920 [85] W. Hong-Xia, "An improved collaborative filtering recommendation algorithm," in IEEE 921 4th Int. Conf. Big Data Anal. (ICBDA), Suzhou, China, 2019, pp. 431-435.

922 [86] G. Chaaya, E. Metais, J. B. Abdo, R. Chiky, J. Demerjian, and K. Barbar, "Evaluating 923 non-personalized single-heuristic active learning strategies for collaborative filtering 924 recommender systems," in $16^{\text {th }}$ IEEE Int. Conf. Mach. Learn. Appl. (ICMLA), Cancun, 2017, pp. 925 593-600.

926 [87] D. R. Shanks, "Is human learning rational?," Q. J. Exp. Psychol. Sect. A: Human 927 Experimental Psychology, vol. 48, no. 2, pp. 257-279, 1995.

928 [88] R. W. Broadley, J. Klenk, S. B. Thies, L. P. J. Kenney, and M. H. Granat, "Methods for 929 the real-world evaluation of fall detection technology: A scoping review," Sensors MDPI, vol.

930 18, no. 7., pp. 1-28, Jun. 2018.

931 [89] P. Pilloni, L. Piras, L. Boratto, S. Carta, G. Fenu, and F. Mulas, "Recommendation in 932 persuasive eHealth systems: An effective strategy to spot users' losing motivation to exercise," 933 in $2^{\text {nd }}$ Int. Works. on Health Rec. Sys., HealthRecSys, 2017.

934 [90] A. Ebadi and A. Krzyzak, "A hybrid multi-criteria hotel recommender system using 935 explicit and implicit feedbacks," Int. J. Sci. Comp. Inf. Eng., vol. 10, no. 8, pp. 1450-1458, 2016. 936 [91] M. A. Marciano, V. R. Williamson, and J. D. Adelman, "A hybrid approach to increase 937 the informedness of CE-based data using locus-specific thresholding and machine learning," 938 Forensic Sci. Int. Genet., vol. 35, no. 1872-4973, pp. 26-37, Jul. 2018. 
939 [92] G. Layher, T. Brosch, and H. Neumann, "Real-time biologically inspired action

940 recognition from key poses using a neuromorphic architecture," Front. Neurorobot., vol. 11, no.

941 13, pp. 1-21, Mar. 2017.

942 [93] S. Boughorbel, F. Jarray, and M. El-Anbari, "Optimal classifier for imbalanced data

943 using Matthews correlation coefficient metric," PLoS One, vol. 12, no. 6, pp. 1-17, Jun. 2017.

944 [94] D. M. W. Powers, "A computationally and cognitively plausible model of supervised and 945 unsupervised learning," in Advan. Brain Insp. Cogn. Sys., 2103, pp. 145-156.

946 [95] K. R. Devi, J. Bhavithra, and A. Saradha, "Diet recommendation for glycemic patients 947 using improved K-means and Krill-Herd optimization," ICTACT J. on S. Comp., vol. 10, no. 03, 948 pp. 2096-2101, Apr. 2020.

949 [96] J. Wang, A. P. De Vries, and M. J. T. Reinders, "Unifying user-based and item-based 950 collaborative filtering approaches by similarity fusion," in the $29^{\text {th }}$ Annu. Int. ACM SIGIR Conf. 951 Res. Dev. Inf. Retr., Seattle, Washington, USA, 2006, pp. 501-508.

952 [97] S. Okyay and S. Aygun, "A Significant Fallacy of Built-in Correlation Functions in 953 Recommender Systems," in 3rd IEEE International Congress on Human-Computer Interaction, 954 Optimization and Robotic Applications. (HORA), June 11-13, 2021.

955 [98] Supriya, “Karl Pearson's Coefficient of Correlation,” Accessed on: Oct. 1, 2021.

956 [Online]. Available: https://www.patnauniversity.ac.in/e-

957 content/social_sciences/geography/MAGeog94.pdf.

958 [99] K. G. Saranya, G. Sudha Sadasivam, and M. Chandralekha, "Performance comparison of 959 different similarity measures for collaborative filtering technique," Indian J. Sci. Technol., pp. 1$9608,2016$. 
Figure 1

Depiction of the paper structure, technical summary, and contributions.

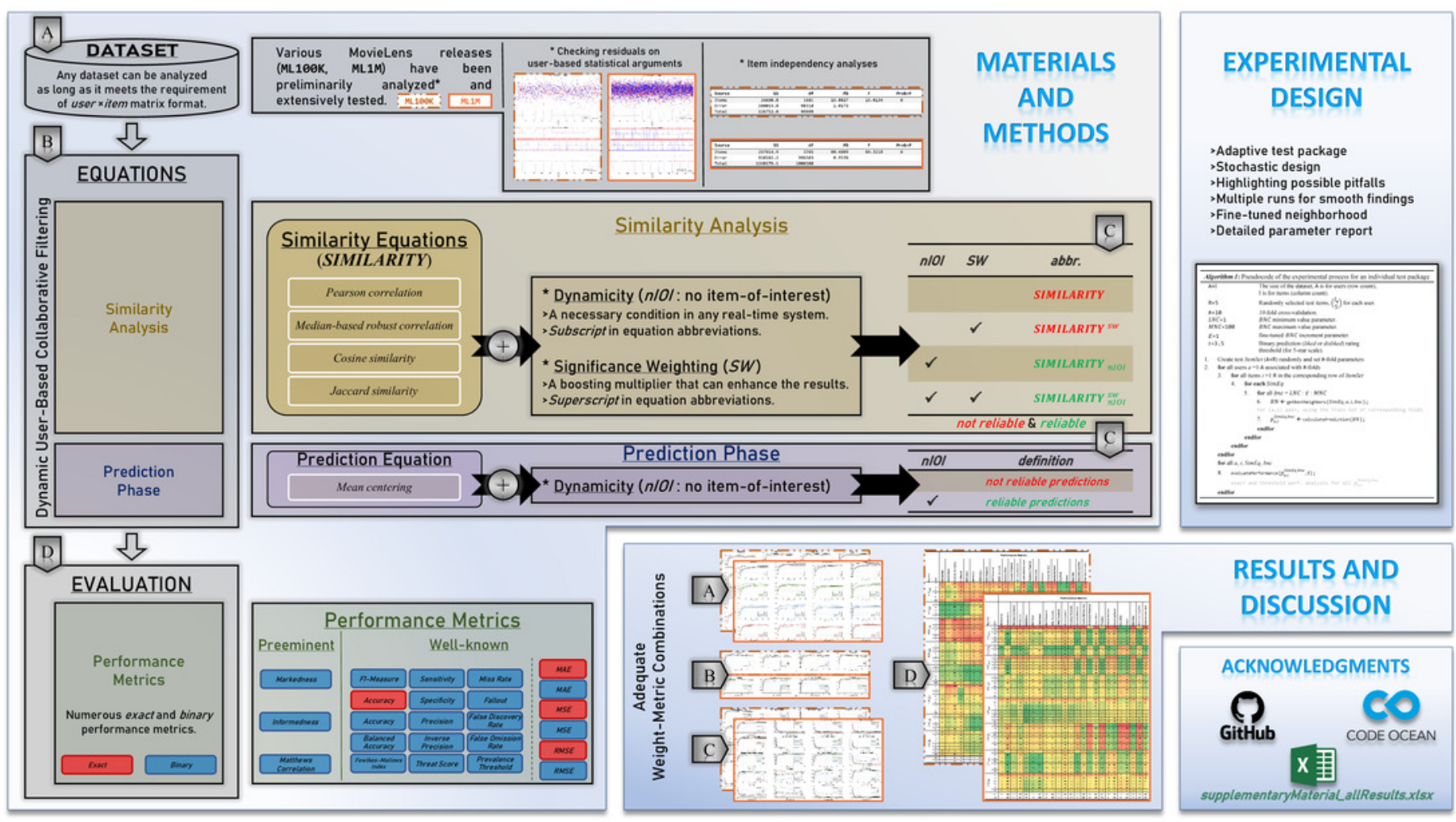




\section{Figure 2}

User-based mean \& median residuals on static vs. dynamic conditions: ML100K. 
Static Mean vs. Dynamic Mean

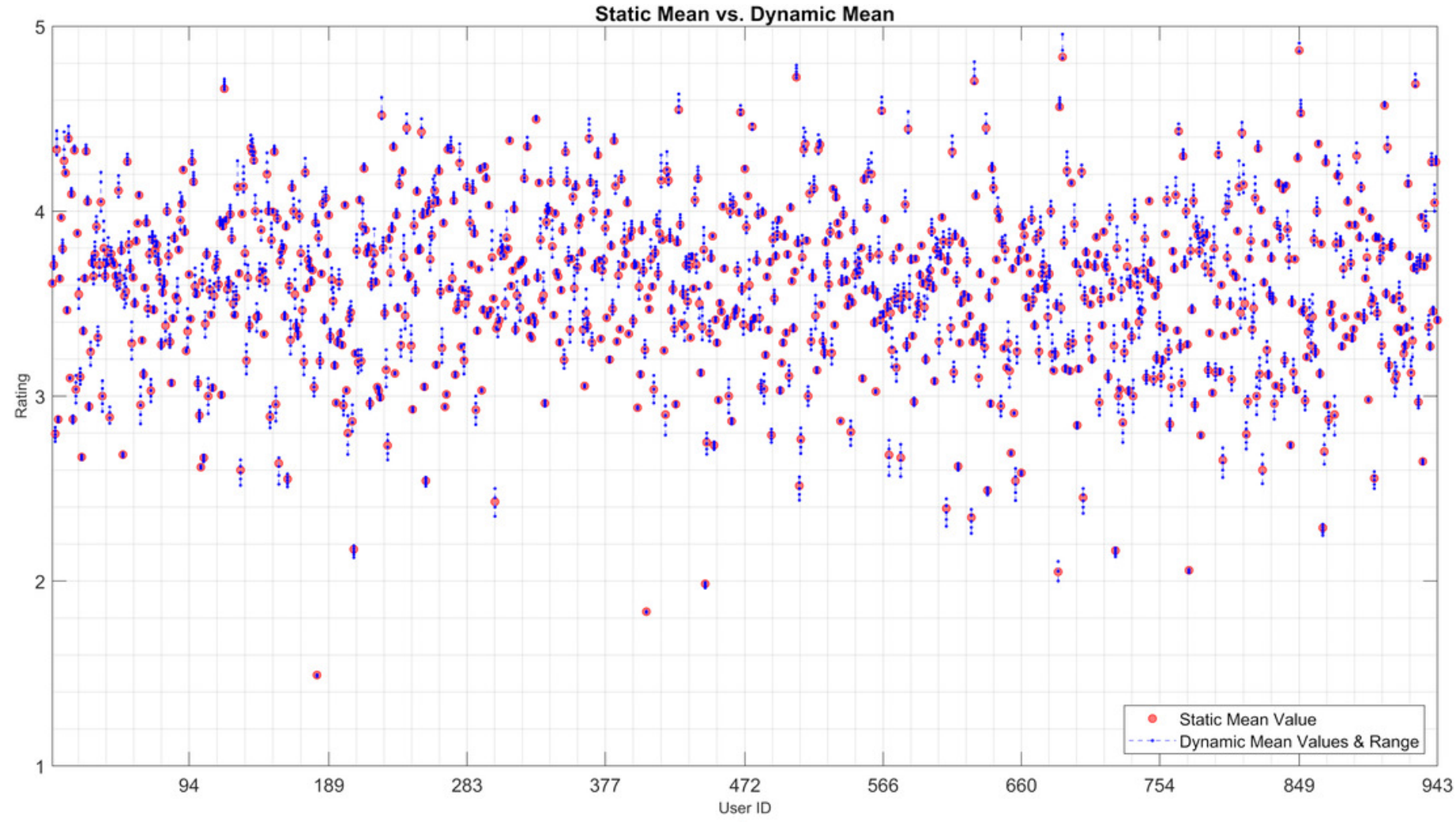

A

Static Median vs. Dynamic Median

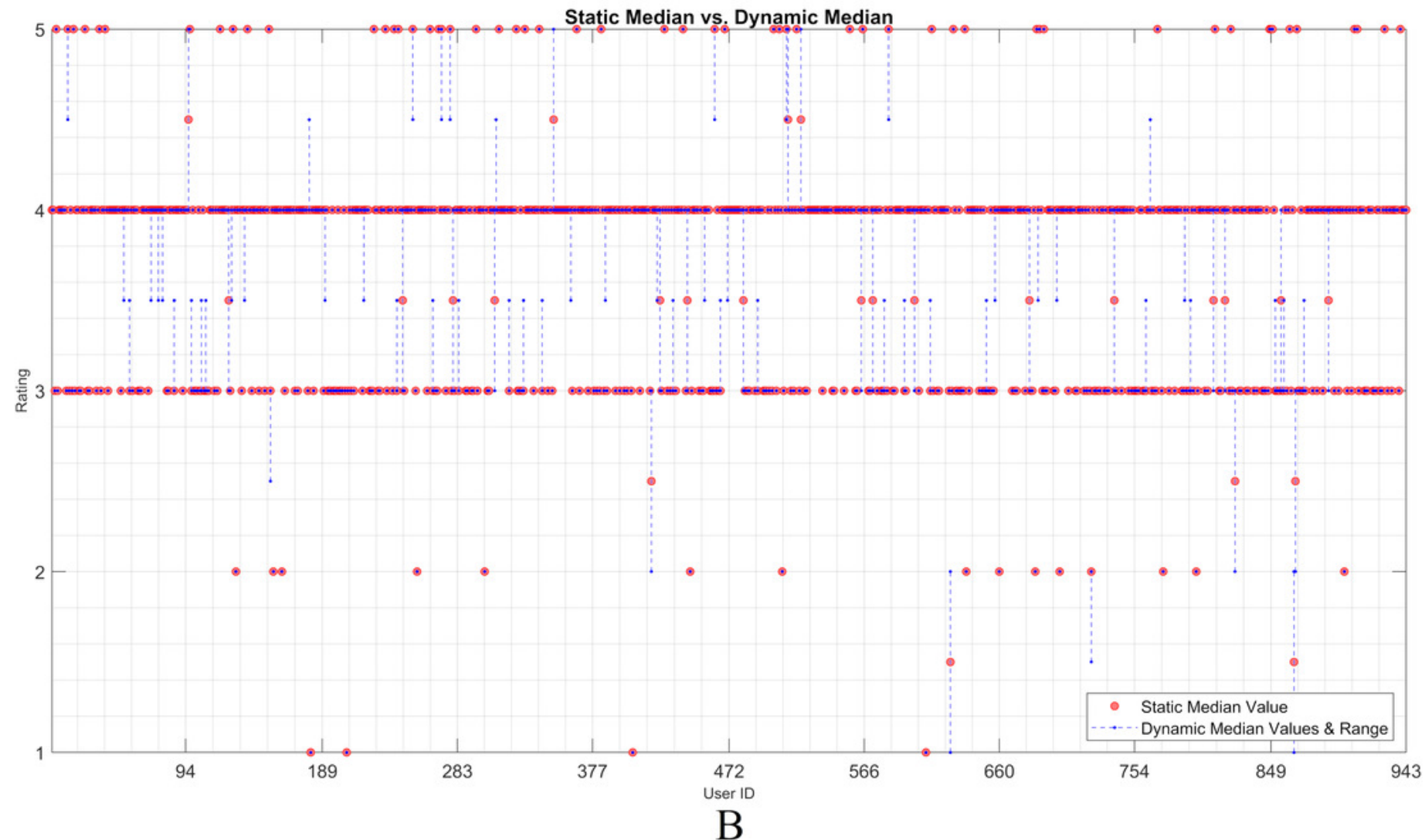




\section{Figure 3}

User-based mean \& median residuals on static vs. dynamic conditions: ML1M. 


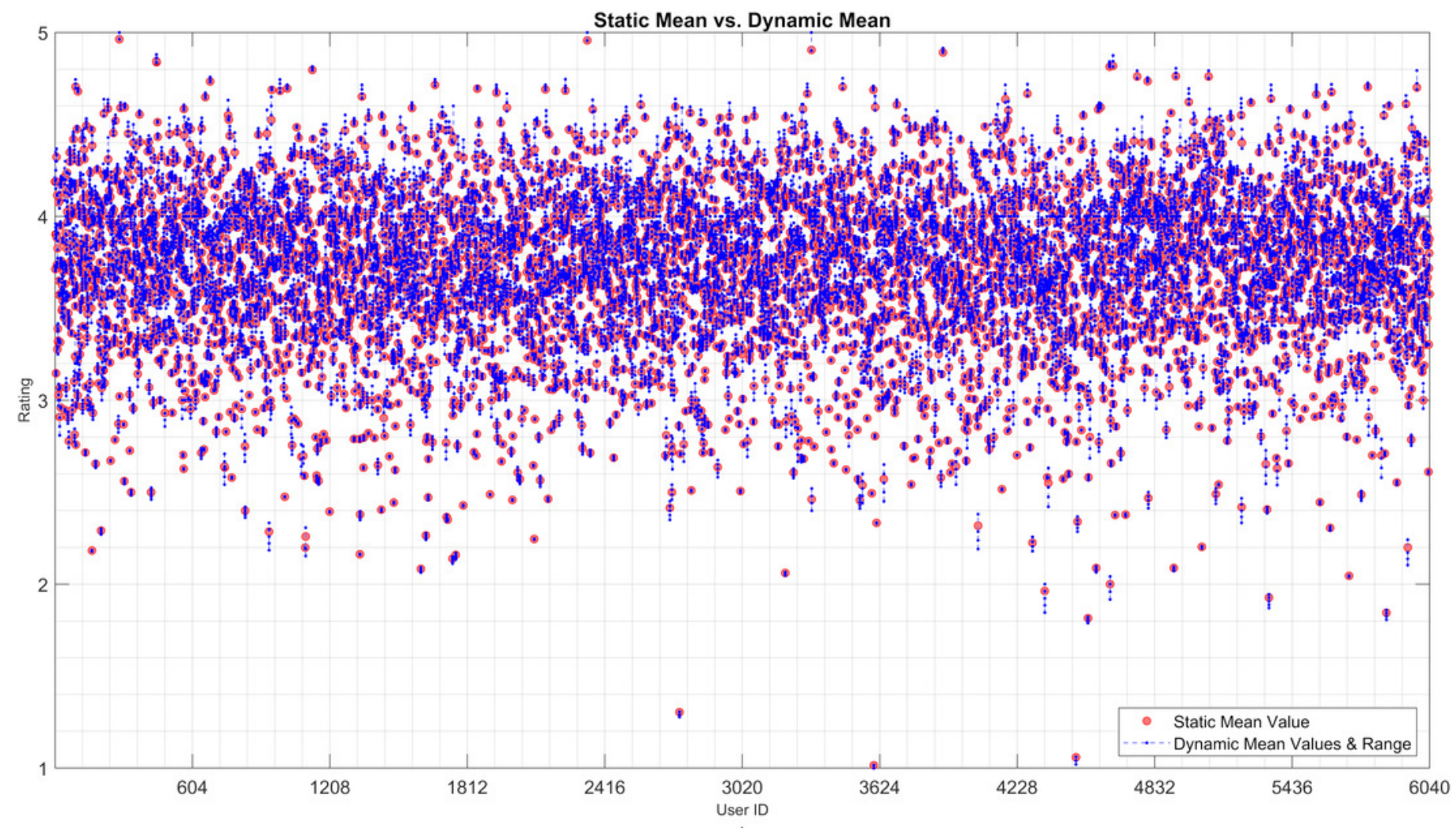

A

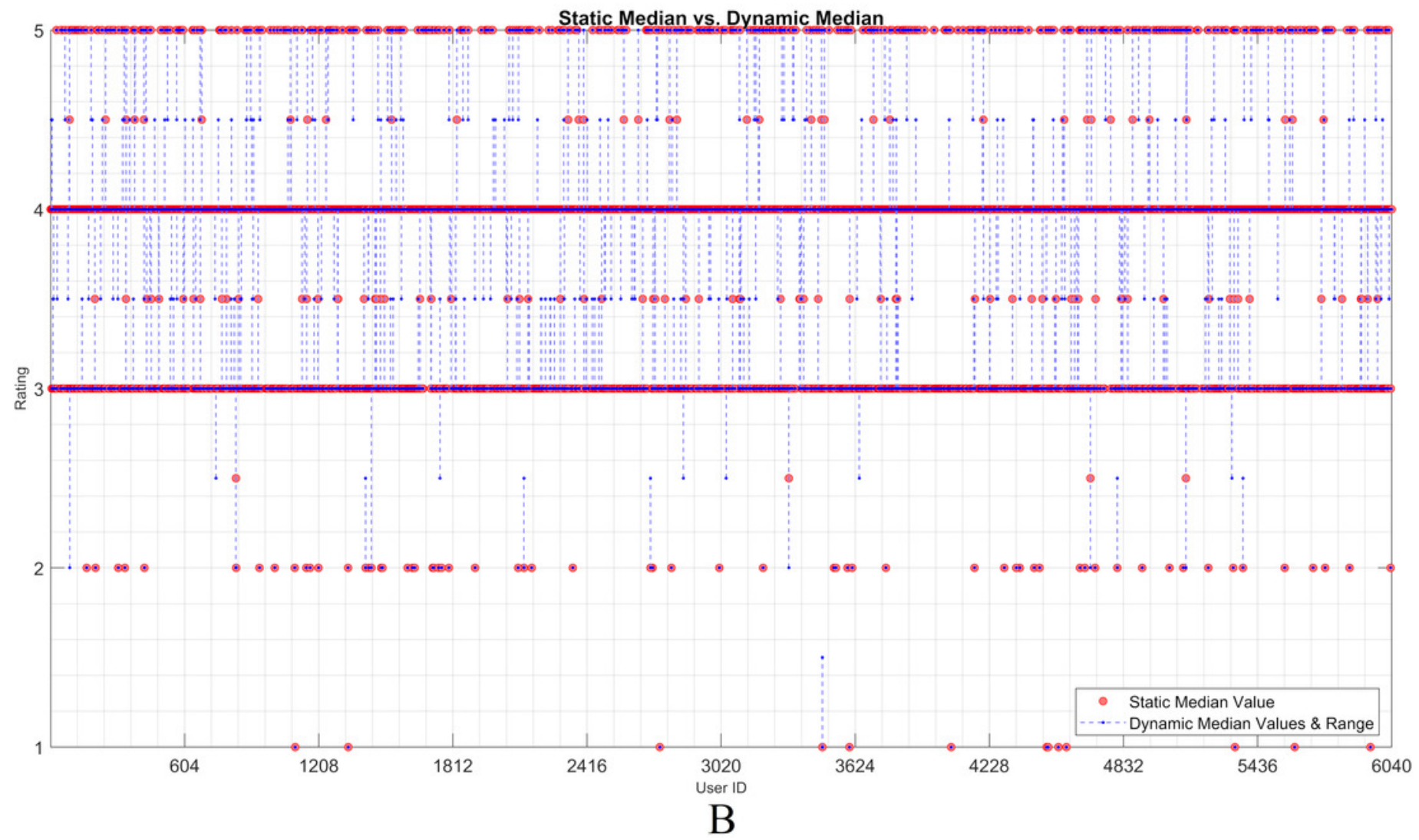


Figure 4

The evaluation over ML100K: similarity weight and preeminent metric combination to compare the dynamicity and weight significance.
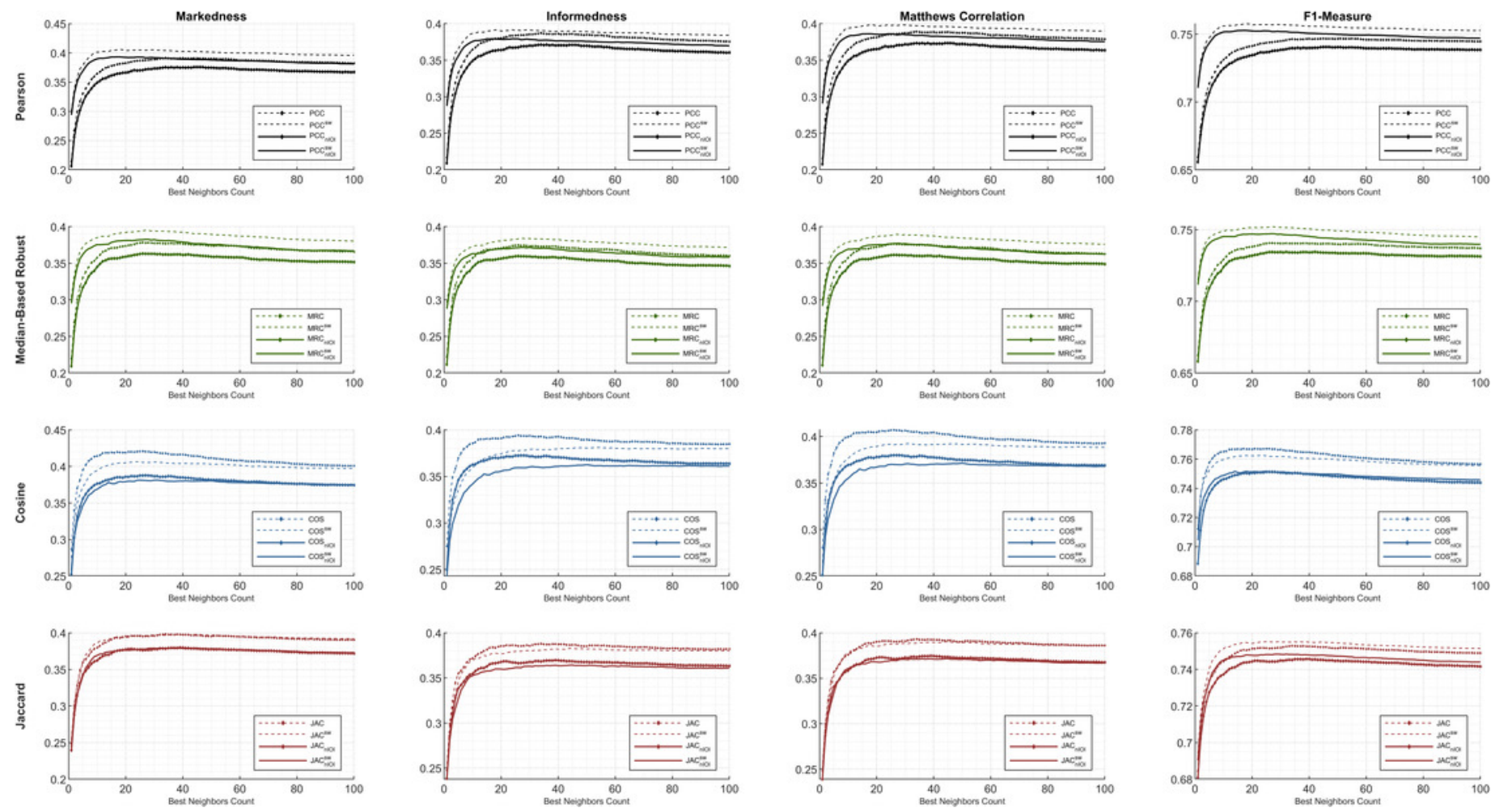
Figure 5

The evaluation over ML1M: similarity weight and preeminent metric combination to compare the dynamicity and weight significance.
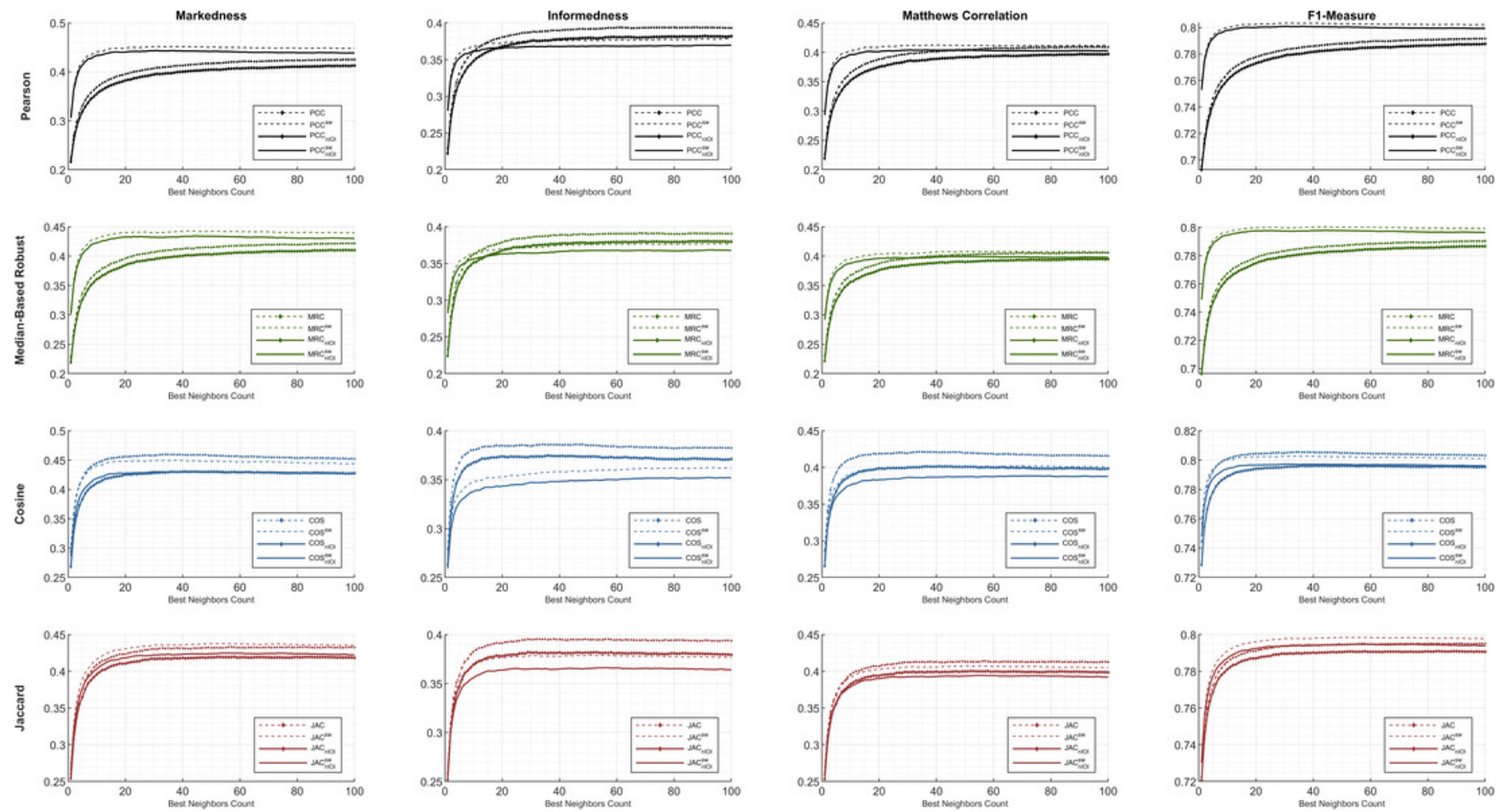
Figure 6

Markedness, informedness, and Matthews correlation as preeminent metrics, plus F1measure highlighting the hybrid monitoring for (a) ML100K and (b) ML1M.
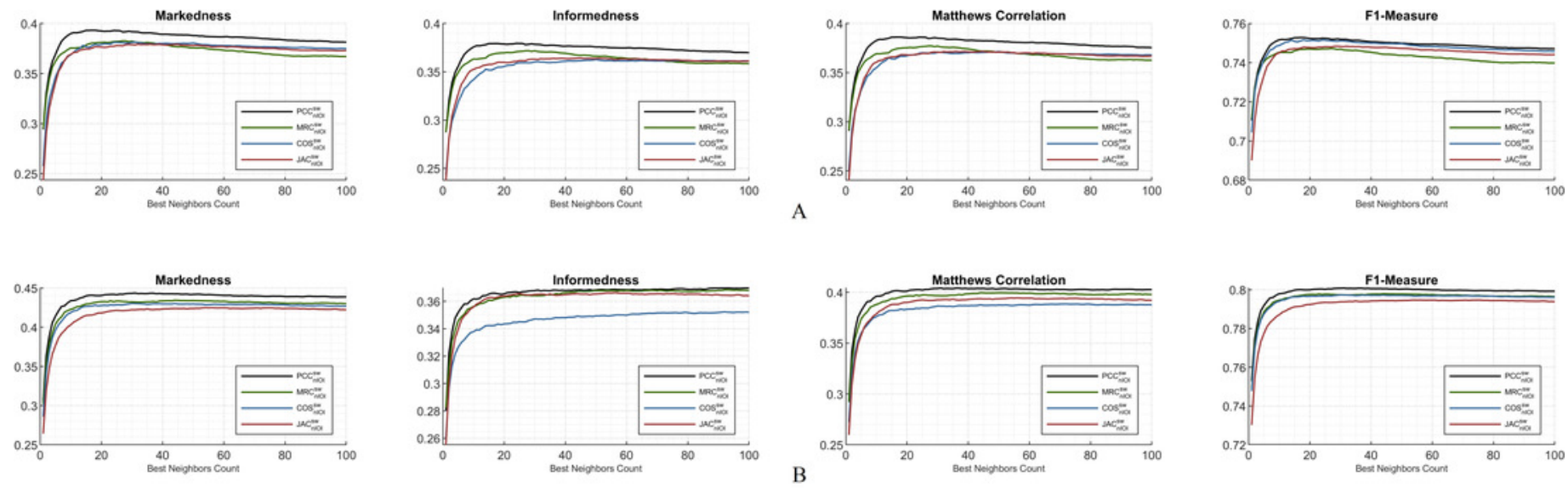
Figure 7

Extended performance evaluation on ML100K considering the hybrid monitoring.
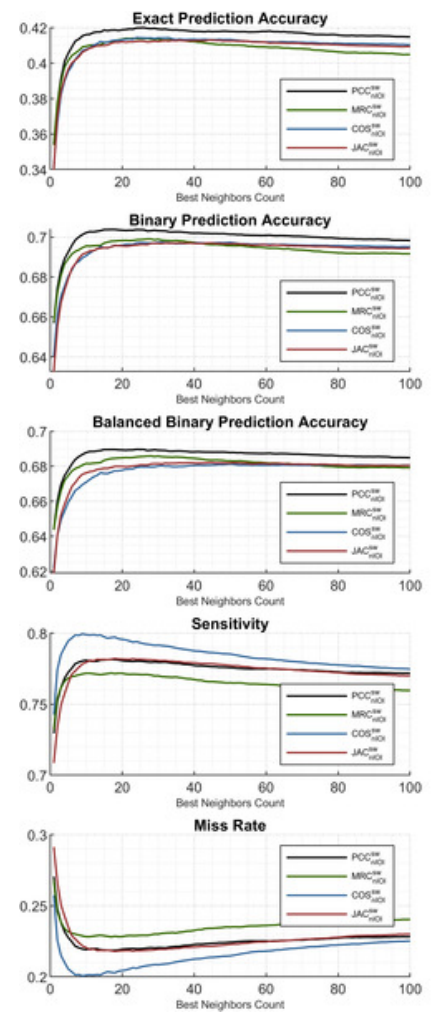
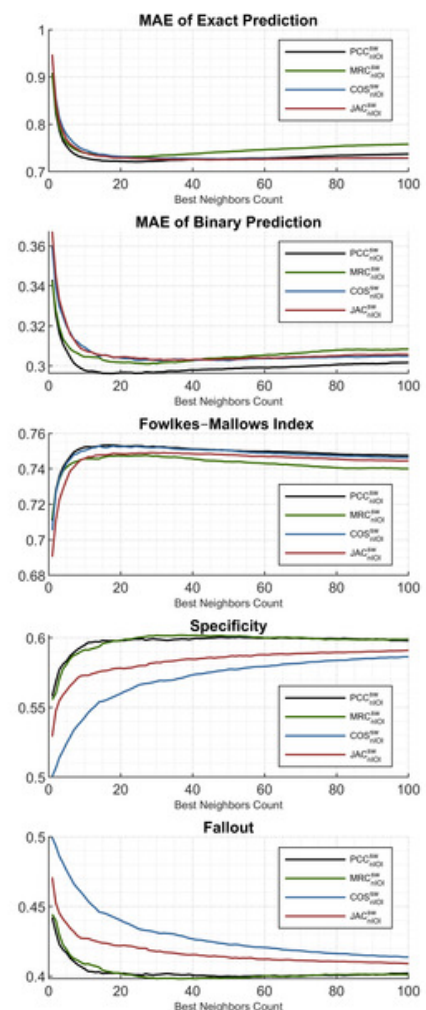
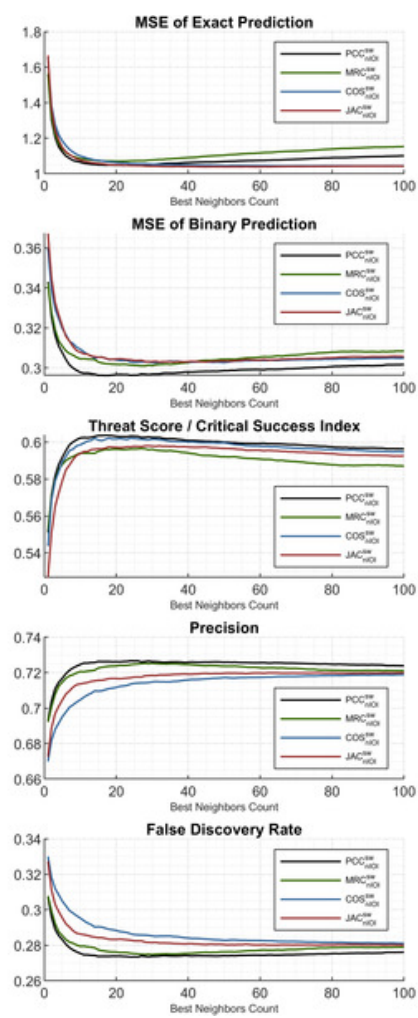
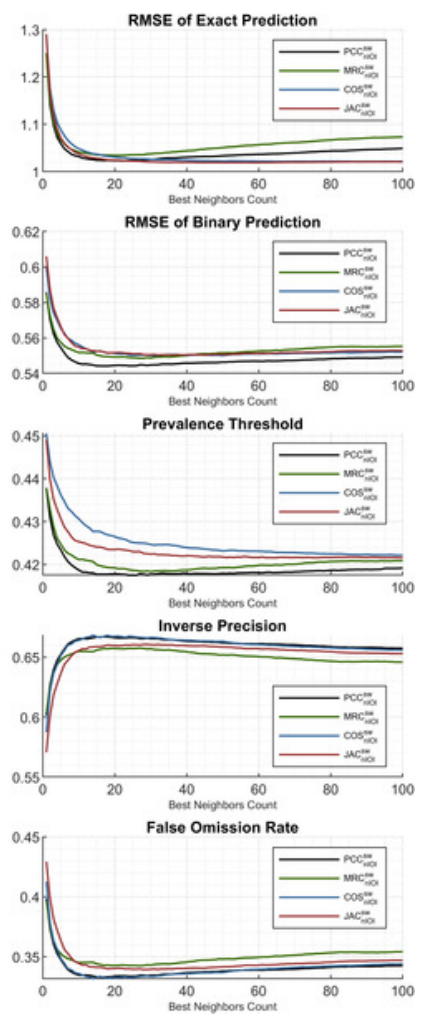
Figure 8

Extended performance evaluation on ML1M considering the hybrid monitoring.
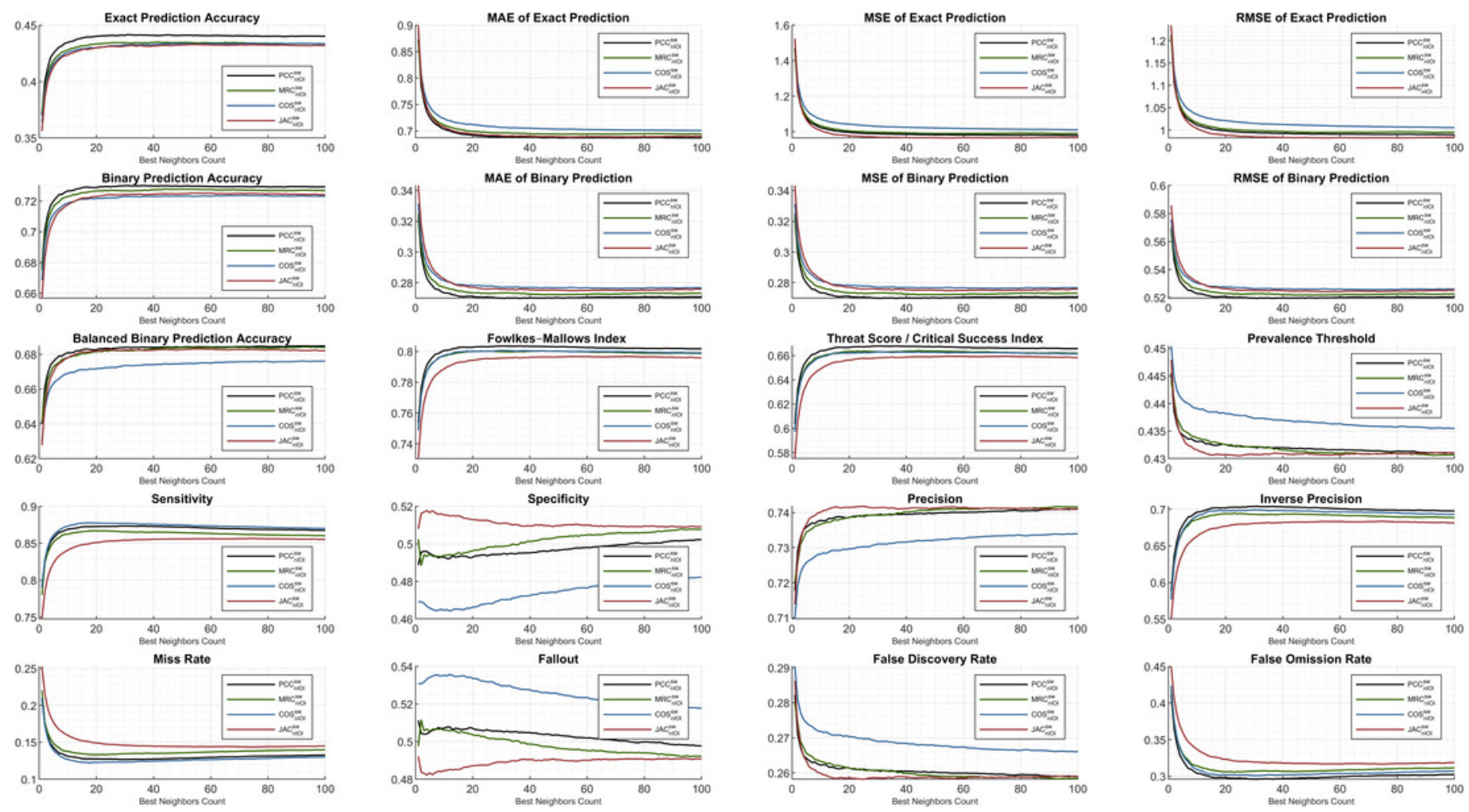


\section{Table $\mathbf{1}$ (on next page)}

Symbols and abbreviations list. 
TABLE 1

SYMBOLS AND ABBREVIATIONS LIST

\begin{tabular}{|c|c|}
\hline $\begin{array}{c}\text { Symbol/ } \\
\text { Abbraviation }\end{array}$ & Explanation \\
\hline $\begin{array}{l}a \\
\hat{a}\end{array}$ & $\begin{array}{l}\text { User-of-interest } \\
\text { User-of-interest where test item bias is discarded }\end{array}$ \\
\hline$i$ & Any item-of-interest \\
\hline$U$ & Possible nominees to be a neighbor \\
\hline $\begin{array}{c}u \\
u^{*}\end{array}$ & $\begin{array}{l}\text { Any possible neighbor for collaboration } \\
\text { Sorted and selected neighbor }\end{array}$ \\
\hline$r_{u}$ & Rating vector of $u$ for all items \\
\hline$r_{u, i}$ & Rating of $u$ for $i$ \\
\hline $\bar{r}_{u}$ & The mean of given ratings for $u$ \\
\hline$\tilde{r}_{u}$ & The median of given ratings for $u$ \\
\hline$I_{u}$ & The rating history of $u$ \\
\hline$P C C$ & Pearson Correlation Coefficient \\
\hline$M R C$ & Median-Based Robust Correlation Coefficient \\
\hline $\cos$ & Cosine Similarity \\
\hline$J A C$ & Jaccard Similarity \\
\hline$w_{a, u}^{S}$ & $\begin{array}{l}\text { Similarity weight between } a \text { and } u \text { for equation } S \text {, } \\
\text { where } S \text { can be given similarity equations }\end{array}$ \\
\hline$p_{a, i}$ & The rating prediction of $a$ for $i$ \\
\hline CIC & Co-rated Item Count between two users \\
\hline$T P$ & True Positive \\
\hline$T N$ & True Negative \\
\hline$F P$ & False Positive \\
\hline$F N$ & False Negative \\
\hline
\end{tabular}




\section{Table 2 (on next page)}

MovieLens (ML) release comparison [9], [27]. 
1

TABLE 2

MOVIELENS (ML) RELEASE COMPARISON [9], [27]

\begin{tabular}{|c|c|c|c|c|c|}
\hline Releases & ML100K & ML1M & ML10M & ML20M & ML25M \\
\hline Number of Ratings & 100,000 & $1,000,209$ & $10,000,054$ & $20,000,263$ & $25,000,095$ \\
\hline Number of Users & 943 & 6,040 & 69,868 & 138,493 & 162,541 \\
\hline Number of Movies & 1,682 & 3,706 & 10,681 & 27,278 & 62,423 \\
\hline Timespan & $09 / 1997-04 / 1998$ & $04 / 2000-02 / 2003$ & $01 / 1995-01 / 2009$ & $01 / 1995-03 / 2015$ & $01 / 1995-11 / 2019$ \\
\hline $\begin{array}{l}\text { Miscellaneous } \\
\text { Information }\end{array}$ & $\begin{array}{l}\text { At least } 20 \text { ratings by } \\
\text { each user, simple } \\
\text { demographic } \\
\text { information for users } \\
\text { (age, gender, } \\
\text { occupation, zip-code). } \\
\text { 5-star rating. }\end{array}$ & $\begin{array}{l}\text { At least } 20 \text { ratings by } \\
\text { each user, simple } \\
\text { demographic } \\
\text { information for users } \\
\text { (age, gender, } \\
\text { occupation, zip-code). } \\
\text { 5-star rating. }\end{array}$ & $\begin{array}{l}\text { At least } 20 \text { ratings by } \\
\text { each user. } \\
\text { No demographic } \\
\text { information. Each user } \\
\text { is represented by only } \\
\text { an ID. } \\
\text { 5-star rating with } \\
\text { half-stars. }\end{array}$ & $\begin{array}{c}\text { At least } 20 \text { ratings } \\
\text { by each user. } \\
\text { No demographic } \\
\text { information. Each user } \\
\text { is represented by only } \\
\text { an ID. } \\
\text { 5-star rating with } \\
\text { half-stars. }\end{array}$ & $\begin{array}{l}\text { At least } 20 \text { ratings } \\
\text { by each user. } \\
\text { No demographic } \\
\text { information. Each user } \\
\text { is represented by only } \\
\text { an ID. } \\
\text { 5-star rating with } \\
\text { half-stars. }\end{array}$ \\
\hline
\end{tabular}




\section{Table 3(on next page)}

ANOVA Table for ML100K. 
TABLE 3

ANOVA TABLE FOR ML100K

\begin{tabular}{lrrrrc}
\hline \hline Source & SS & df & MS & F & Prob $>$ F \\
\hline Items & 26698.8 & 1681 & 15.8827 & 15.6134 & 0 \\
Error & 100014.0 & 98318 & 1.0173 & & \\
Total & 126712.8 & 99999 & & & \\
\hline \hline
\end{tabular}




\section{Table 4(on next page)}

ANOVA Table for ML1M. 
TABLE 4

ANOVA TABLE FOR ML1M

\begin{tabular}{lrrrrc}
\hline \hline Source & SS & df & MS & F & Prob>F \\
\hline Items & 297914.9 & 3705 & 80.4089 & 84.3218 & 0 \\
Error & 950261.2 & 996503 & 0.9536 & & \\
Total & 1248176.1 & 1000208 & & & \\
\hline \hline
\end{tabular}




\section{Table 5 (on next page)}

Well-known performance metrics. 
TABLE 5

WELL-KNOWN PERFORMANCE METRICS

\begin{tabular}{|c|c|}
\hline Metric Name & Formula \\
\hline Exact Accuracy & $\frac{\text { Exact Prediction Count }}{T P+T N+F P+F N}$ \\
\hline Threshold Accuracy & $\frac{T P+T N}{T P+T N+F P+F N}$ \\
\hline $\begin{array}{c}\text { Sensitivity/ } \\
\text { Recall/ } \\
\text { True Positive Rate }\end{array}$ & $\frac{T P}{T P+F N}$ \\
\hline $\begin{array}{l}\text { Precision / } \\
\text { Positive Pred. Value }\end{array}$ & $\frac{T P}{T P+F P}$ \\
\hline F1-Measure & $\frac{2 \times T P}{2 \times T P+F P+F N}$ \\
\hline $\begin{array}{c}\text { Specificity / } \\
\text { Inverse Sensitivity/ } \\
\text { True Negative Rate }\end{array}$ & $\frac{T N}{F P+T N}$ \\
\hline $\begin{array}{l}\text { Inverse Precision / } \\
\text { Negative Pred. Value }\end{array}$ & $\frac{T N}{T N+F N}$ \\
\hline False Discovery Rate & $1-$ Precision \\
\hline False Omission Rate & 1 -Inverse Precision \\
\hline $\begin{array}{c}\text { Fallout/ } \\
\text { False Positive Rate }\end{array}$ & $1-$ Specificity \\
\hline $\begin{array}{l}\text { Miss Rate/ } \\
\text { False Negative Rate }\end{array}$ & $1-$ Sensitivity \\
\hline $\begin{array}{l}\text { Fowlkes-Mallows } \\
\text { Index }\end{array}$ & $\sqrt{\text { Precision } \times \text { Sensitivity }}$ \\
\hline Balanced Accuracy & $\frac{(\text { Sensitivity }+ \text { Specificity })}{2}$ \\
\hline $\begin{array}{l}\text { Threat Score/ } \\
\text { Critical Success Index }\end{array}$ & $\frac{T P}{T P+F N+F P}$ \\
\hline Prevalence Threshold & $\frac{\sqrt{\text { Sensitivity } \times(1-\text { Specificity })}+\text { Specificity }-1}{\text { Sensitivity }+ \text { Specificity }-1}$ \\
\hline
\end{tabular}




\section{Table 6(on next page)}

Preeminent performance metrics. 
TABLE 6

PREEMINENT PERFORMANCE METRICS

\begin{tabular}{cc}
\hline \hline Metric Name & Formula \\
\hline Markedness & $\frac{T P}{T P+F P}+\frac{T N}{T N+F N}-1$ \\
Informedness & $\frac{T P}{T P+F N}+\frac{T N}{T N+F P}-1$ \\
$\begin{array}{c}\text { Matthews } \\
\text { Correlation }\end{array}$ & $\frac{T P \times T N-F P \times F N}{\sqrt{(T P+F P) \times(T P+F N) \times(T N+F P) \times(T N+F N)}}$ \\
\hline \hline
\end{tabular}




\section{Table 7 (on next page)}

All test configurations considering $\mathrm{nIOI}$ and $\mathrm{SW}$ similarity measurements. 
TABLE 7

ALl Test CONFIGURATIONS CONSIDERING $n$ IOI AND $S W$ Similarity MEASUREMENTS

\begin{tabular}{|c|c|c|c|c|}
\hline $\begin{array}{c}\text { Abbreviation of } \\
\text { Similarity } \\
\text { Equation }\end{array}$ & Dynamic & $\begin{array}{c}\text { Significance } \\
\text { Weighting }\end{array}$ & $\begin{array}{c}\text { Related } \\
\text { Equation }\end{array}$ & $\begin{array}{c}\boldsymbol{B N C} \\
L N C: \mathcal{E}: M N C\end{array}$ \\
\hline$P C C$ & & & Eq. (1) & \multirow{16}{*}{$1: 1: 100$} \\
\hline$P C C^{S W}$ & & $\checkmark$ & Eq. (7) & \\
\hline$P C C_{n I O I}$ & $\checkmark$ & & Eq. (6) & \\
\hline$P C C_{n I O I}^{s w}$ & $\checkmark$ & $\checkmark$ & Eq. (14) & \\
\hline$M R C$ & & & Eq. (2) & \\
\hline$M R C^{s w}$ & & $\checkmark$ & Eq. (9) & \\
\hline$M R C_{n I O I}$ & $\checkmark$ & & Eq. (8) & \\
\hline$M R C_{n I O I}^{s w}$ & $\checkmark$ & $\checkmark$ & Eq. (14) & \\
\hline $\cos$ & & & Eq. (3) & \\
\hline $\cos ^{s w}$ & & $\checkmark$ & Eq. (11) & \\
\hline $\operatorname{COS}_{\text {nIOI }}$ & $\checkmark$ & & Eq. (10) & \\
\hline $\operatorname{COS}_{n I O I}^{s W}$ & $\checkmark$ & $\checkmark$ & Eq. (14) & \\
\hline$J A C$ & & & Eq. (4) & \\
\hline$J A C^{S w}$ & & $\checkmark$ & Eq. (13) & \\
\hline$J A C_{n I O I}$ & $\checkmark$ & & Eq. (12) & \\
\hline$J A C_{n I O I}^{s W}$ & $\checkmark$ & $\checkmark$ & Eq. (14) & \\
\hline
\end{tabular}




\section{Table 8(on next page)}

The best-performing BNC values under a variety of performance metrics: ML100K. 
TABLE 8

The Best-Performing $B N C$ VAlues Under A VARIETy of PerformanCe Metrics: ML100K

\begin{tabular}{|c|c|c|c|c|c|c|c|c|c|c|c|c|c|c|c|c|c|c|c|c|c|c|c|c|}
\hline & \multicolumn{24}{|c|}{ Performance Metrics } \\
\hline Equation & 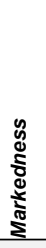 & 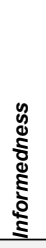 & 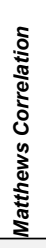 & 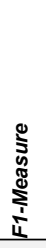 & 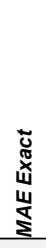 & 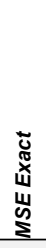 & 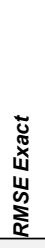 & 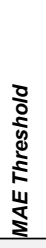 & 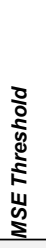 & 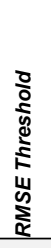 & 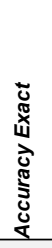 & 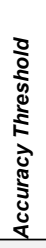 & 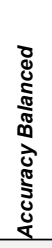 & 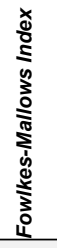 & 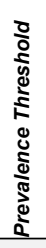 & 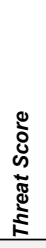 & 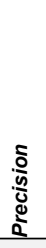 & 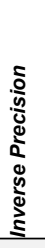 & 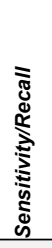 & 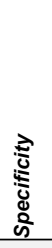 & 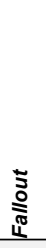 & 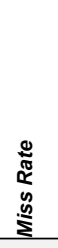 & 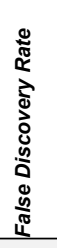 & 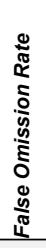 \\
\hline$P C C_{n I O I}$ & 45 & 34 & 45 & 47 & 34 & 23 & 23 & 45 & 45 & 45 & 34 & 45 & 34 & 47 & 34 & 47 & 34 & 47 & 100 & 17 & 17 & 100 & 34 & 47 \\
\hline$P C C_{n I O I}^{s w}$ & 17 & 24 & 17 & 17 & 24 & 22 & 22 & 17 & 17 & 17 & 26 & 17 & 24 & 17 & 26 & 17 & 26 & 17 & 17 & 53 & 53 & 17 & 26 & 17 \\
\hline$\downarrow / \uparrow$ & $\downarrow$ & $\downarrow$ & $\downarrow$ & $\downarrow$ & $\downarrow$ & $\downarrow$ & $\downarrow$ & $\downarrow$ & $\downarrow$ & $\downarrow$ & $\downarrow$ & $\downarrow$ & $\downarrow$ & $\downarrow$ & $\downarrow$ & $\downarrow$ & $\downarrow$ & $\downarrow$ & $\downarrow$ & $\uparrow$ & $\uparrow$ & $\downarrow$ & $\downarrow$ & $\downarrow$ \\
\hline$M R C_{n I O I}$ & 26 & 26 & 26 & 42 & 27 & 19 & 19 & 26 & 26 & 26 & 27 & 26 & 26 & 42 & 25 & 42 & 25 & 42 & 59 & 12 & 12 & 59 & 25 & 42 \\
\hline$M R C_{n I O I}^{s w}$ & 27 & 28 & 28 & 27 & 23 & 20 & 20 & 27 & 27 & 27 & 23 & 27 & 28 & 27 & 30 & 27 & 30 & 27 & 17 & 37 & 37 & 17 & 30 & 27 \\
\hline$\downarrow / \uparrow$ & $\uparrow$ & $\uparrow$ & $\uparrow$ & $\downarrow$ & $\downarrow$ & $\uparrow$ & $\uparrow$ & $\uparrow$ & $\uparrow$ & $\uparrow$ & $\downarrow$ & $\uparrow$ & $\uparrow$ & $\downarrow$ & $\uparrow$ & $\downarrow$ & $\uparrow$ & $\downarrow$ & $\downarrow$ & $\uparrow$ & $\uparrow$ & $\downarrow$ & $\uparrow$ & $\downarrow$ \\
\hline $\operatorname{COS}_{n I O I}$ & 27 & 27 & 27 & 25 & 28 & 35 & 35 & 27 & 27 & 27 & 27 & 27 & 27 & 25 & 36 & 25 & 36 & 25 & 25 & 100 & 100 & 25 & 36 & 25 \\
\hline $\operatorname{COS}_{n I O I}^{S W}$ & 31 & 50 & 50 & 18 & 50 & 67 & 67 & 31 & 31 & 31 & 24 & 31 & 50 & 14 & 99 & 18 & 99 & 14 & 9 & 100 & 100 & 9 & 99 & 14 \\
\hline$\downarrow / \uparrow$ & $\uparrow$ & $\uparrow$ & $\uparrow$ & $\downarrow$ & $\uparrow$ & $\uparrow$ & $\uparrow$ & $\uparrow$ & $\uparrow$ & $\uparrow$ & $\downarrow$ & $\uparrow$ & $\uparrow$ & $\downarrow$ & $\uparrow$ & $\downarrow$ & $\uparrow$ & $\downarrow$ & $\downarrow$ & $\Leftrightarrow$ & $\Leftrightarrow$ & $\downarrow$ & $\uparrow$ & $\downarrow$ \\
\hline$J A C_{n I O I}$ & 39 & 39 & 39 & 39 & 40 & 40 & 40 & 39 & 39 & 39 & 36 & 39 & 39 & 39 & 39 & 39 & 39 & 39 & 35 & 20 & 20 & 35 & 39 & 39 \\
\hline$J A C_{n I O I}^{S W}$ & 32 & 45 & 45 & 29 & 45 & 59 & 59 & 36 & 36 & 36 & 44 & 36 & 45 & 29 & 100 & 29 & 100 & 29 & 18 & 100 & 100 & 18 & 100 & 29 \\
\hline$\downarrow / \uparrow$ & $\downarrow$ & $\uparrow$ & $\uparrow$ & $\downarrow$ & $\uparrow$ & $\uparrow$ & $\uparrow$ & $\downarrow$ & $\downarrow$ & $\downarrow$ & $\uparrow$ & $\downarrow$ & $\uparrow$ & $\downarrow$ & $\uparrow$ & $\downarrow$ & $\uparrow$ & $\downarrow$ & $\downarrow$ & $\uparrow$ & $\uparrow$ & $\downarrow$ & $\uparrow$ & $\downarrow$ \\
\hline
\end{tabular}




\section{Table 9 (on next page)}

The best-performing BNC values under a variety of performance metrics: ML1M. 
TABLE 9

The Best-Performing $B N C$ Values Under a Variety of Performance Metrics: ML1M

\begin{tabular}{|c|c|c|c|c|c|c|c|c|c|c|c|c|c|c|c|c|c|c|c|c|c|c|c|c|}
\hline \multirow[b]{2}{*}{ Equation } & \multicolumn{24}{|c|}{ Performance Metrics } \\
\hline & 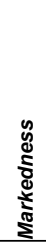 & 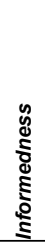 & 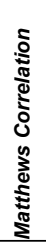 & 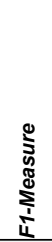 & 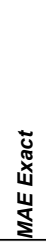 & 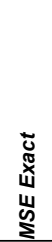 & 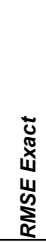 & 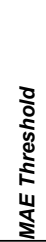 & 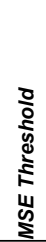 & 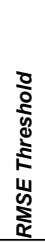 & 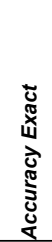 & 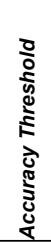 & 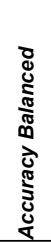 & 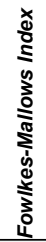 & 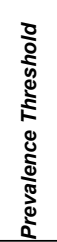 & 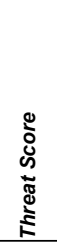 & 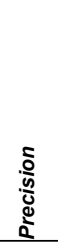 & 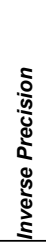 & 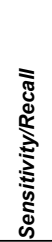 & 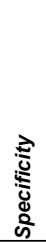 & 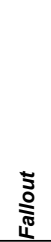 & 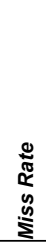 & 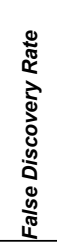 & 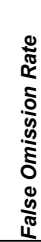 \\
\hline$P C C_{n I O I}$ & 100 & 91 & 91 & 100 & 99 & 91 & 91 & 100 & 100 & 100 & 96 & 100 & 91 & 100 & 31 & 100 & 31 & 100 & 100 & 4 & 4 & 100 & 31 & 100 \\
\hline$P C C_{n I O I}^{s w}$ & 31 & 93 & 31 & 31 & 100 & 100 & 100 & 31 & 31 & 31 & 31 & 31 & 93 & 31 & 100 & 31 & 100 & 31 & 31 & 100 & 100 & 31 & 100 & 31 \\
\hline$\downarrow / \uparrow$ & $\downarrow$ & $\uparrow$ & $\downarrow$ & $\downarrow$ & $\uparrow$ & $\uparrow$ & $\uparrow$ & $\downarrow$ & $\downarrow$ & $\downarrow$ & $\downarrow$ & $\downarrow$ & $\uparrow$ & $\downarrow$ & $\uparrow$ & $\downarrow$ & $\uparrow$ & $\downarrow$ & $\downarrow$ & $\uparrow$ & $\uparrow$ & $\downarrow$ & $\uparrow$ & $\downarrow$ \\
\hline$M R C_{n I O I}$ & 97 & 92 & 92 & 97 & 97 & 60 & 60 & 92 & 92 & 92 & 92 & 92 & 92 & 97 & 48 & 97 & 48 & 97 & 100 & 8 & 8 & 100 & 48 & 97 \\
\hline$M R C_{n I O I}^{s w}$ & 44 & 93 & 44 & 44 & 58 & 94 & 94 & 44 & 44 & 44 & 41 & 44 & 93 & 44 & 93 & 44 & 93 & 23 & 23 & 96 & 96 & 23 & 93 & 23 \\
\hline$\downarrow / \uparrow$ & $\downarrow$ & $\uparrow$ & $\downarrow$ & $\downarrow$ & $\downarrow$ & $\uparrow$ & $\uparrow$ & $\downarrow$ & $\downarrow$ & $\downarrow$ & $\downarrow$ & $\downarrow$ & $\uparrow$ & $\downarrow$ & $\uparrow$ & $\downarrow$ & $\uparrow$ & $\downarrow$ & $\downarrow$ & $\uparrow$ & $\uparrow$ & $\downarrow$ & $\uparrow$ & $\downarrow$ \\
\hline $\operatorname{COS}_{\text {nIOI }}$ & 41 & 36 & 41 & 41 & 45 & 45 & 45 & 41 & 41 & 41 & 72 & 41 & 36 & 50 & 13 & 41 & 13 & 50 & 83 & 4 & 4 & 83 & 13 & 50 \\
\hline $\cos _{n I O I}^{s w}$ & 30 & 92 & 72 & 30 & 97 & 100 & 100 & 72 & 72 & 72 & 91 & 72 & 92 & 30 & 99 & 30 & 99 & 30 & 18 & 99 & 99 & 18 & 99 & 30 \\
\hline$\downarrow / \uparrow$ & $\downarrow$ & $\uparrow$ & $\uparrow$ & $\downarrow$ & $\uparrow$ & $\uparrow$ & $\uparrow$ & $\uparrow$ & $\uparrow$ & $\uparrow$ & $\uparrow$ & $\uparrow$ & $\uparrow$ & $\downarrow$ & $\uparrow$ & $\downarrow$ & $\uparrow$ & $\downarrow$ & $\downarrow$ & $\uparrow$ & $\uparrow$ & $\downarrow$ & $\uparrow$ & $\downarrow$ \\
\hline$J A C_{n I O I}$ & 58 & 29 & 58 & 84 & 58 & 53 & 53 & 58 & 58 & 58 & 58 & 58 & 29 & 84 & 29 & 84 & 29 & 90 & 99 & 10 & 10 & 99 & 29 & 90 \\
\hline$J A C_{n I O I}^{s w}$ & 54 & 56 & 56 & 54 & 55 & 55 & 55 & 56 & 56 & 56 & 54 & 56 & 56 & 75 & 25 & 54 & 25 & 75 & 75 & 4 & 4 & 75 & 25 & 75 \\
\hline$\downarrow / \uparrow$ & $\downarrow$ & $\uparrow$ & $\downarrow$ & $\downarrow$ & $\downarrow$ & $\uparrow$ & $\uparrow$ & $\downarrow$ & $\downarrow$ & $\downarrow$ & $\downarrow$ & $\downarrow$ & $\uparrow$ & $\downarrow$ & $\downarrow$ & $\downarrow$ & $\downarrow$ & $\downarrow$ & $\downarrow$ & $\downarrow$ & $\downarrow$ & $\downarrow$ & $\downarrow$ & $\downarrow$ \\
\hline
\end{tabular}




\section{Table $\mathbf{1 0}$ (on next page)}

Adequate weight-metric combination of the top-performing BNCs: ML100K. 
TABLE 10

Adequate Weight-Metric COMBination of THe Top-Performing $B N C_{\mathrm{s}}$ : ML100K

Performance Metrics

\begin{tabular}{|c|c|c|c|c|c|c|c|c|c|c|c|c|c|c|c|c|c|c|c|c|c|c|c|c|c|}
\hline \multirow[b]{2}{*}{ 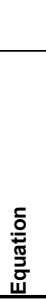 } & \multirow[b]{2}{*}{$z_{0}^{u}$} & \multicolumn{24}{|c|}{ Performance Metrics } \\
\hline & & 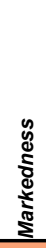 & 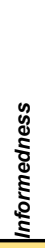 & 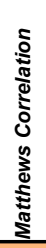 & 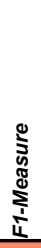 & 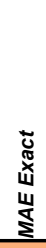 & 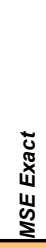 & 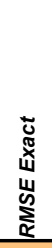 & 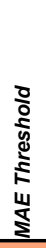 & 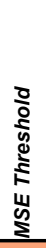 & 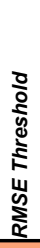 & 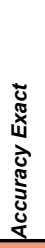 & 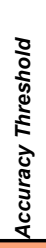 & 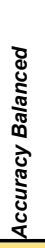 & 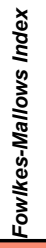 & 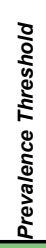 & 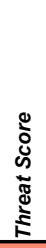 & 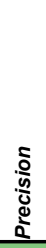 & 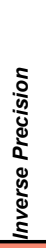 & 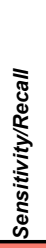 & 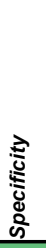 & 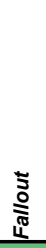 & 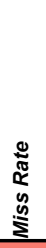 & 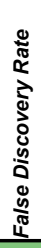 & 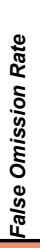 \\
\hline \multirow{6}{*}{ 衣 } & 17 & .364 & .363 & .364 & .733 & .744 & 1.081 & 1.040 & .310 & .310 & .557 & .403 & .690 & .681 & .733 & .416 & .579 & .729 & $\begin{array}{l}.635 \\
\end{array}$ & .737 & .626 & .374 & .263 & .271 & .365 \\
\hline & 23 & .371 & .368 & .369 & .737 & .740 & 1.075 & 1.037 & .307 & .307 & .554 & .405 & .693 & .684 & .737 & .416 & .583 & .730 & .641 & .744 & $\begin{array}{l}.624 \\
\end{array}$ & .376 & .256 & 270 & .359 \\
\hline & 34 & .375 & .371 & .373 & .740 & .739 & 1.078 & 1.038 & .304 & .304 & .552 & .407 & .696 & .686 & .740 & .415 & .587 & .730 & .645 & .750 & .622 & .378 & .250 & 270 & .355 \\
\hline & 45 & .376 & .371 & .374 & .741 & .742 & 1.091 & 1.044 & .304 & .304 & .551 & .406 & .696 & .686 & .741 & .416 & .588 & .729 & .647 & .752 & .619 & .381 & .248 & .271 & .353 \\
\hline & 47 & .376 & .371 & .373 & .741 & .742 & 1.092 & 1.045 & .304 & .304 & .552 & .406 & .696 & .685 & .741 & .416 & .588 & .729 & .647 & .753 & .618 & .382 & .247 & .271 & .353 \\
\hline & 100 & .367 & .360 & .364 & .739 & .756 & 1.141 & 1.068 & .308 & .308 & .555 & .403 & .692 & .680 & .739 & .419 & .586 & .723 & .644 & .754 & .606 & .394 & 246 & .277 & .356 \\
\hline \multirow{5}{*}{$\begin{array}{l}\text { s. } \\
\text { yु } \\
\text { हु }\end{array}$} & 17 & .393 & .379 & .3866 & .753 & .722 & 1.048 & 1.023 & 296 & 296 & .544 & .419 & .704 & .690 & .753 & .418 & .604 & .726 & .667 & .781 & .598 & .402 & .219 & .274 & .333 \\
\hline & 22 & .392 & .379 & .385 & .752 & .721 & 1.046 & 1.023 & 297 & 297 & .545 & .419 & .703 & .689 & .753 & .418 & .603 & .726 & .666 & .780 & .598 & .402 & .220 & .274 & .334 \\
\hline & 24 & .393 & .379 & .386 & .753 & .721 & 1.047 & 1.023 & 296 & 296 & .544 & .420 & .704 & .690 & .753 & .417 & .603 & .727 & .666 & .780 & .599 & .401 & .220 & .273 & .334 \\
\hline & 26 & .393 & .379 & $\begin{array}{l}.386 \\
\end{array}$ & .752 & .721 & 1.049 & 1.024 & 296 & .296 & .544 & .420 & .704 & .690 & .753 & .417 & .603 & .727 & .666 & .780 & .599 & .401 & .220 & 273 & .334 \\
\hline & 53 & .388 & .376 & .382 & .750 & .728 & 1.070 & 1.034 & .299 & .299 & .547 & .418 & .701 & .688 & .750 & .418 & .600 & .726 & .662 & .775 & .600 & .400 & .225 & 274 & .338 \\
\hline \multirow{7}{*}{ 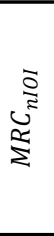 } & 12 & .353 & .351 & .352 & .728 & .755 & 1.105 & 1.051 & .316 & .316 & .562 & .397 & .684 & .676 & .728 & .419 & .572 & .724 & .628 & .732 & .620 & .380 & 268 & .276 & .372 \\
\hline & 19 & .358 & .356 & .357 & .732 & .750 & 1.096 & 1.047 & .313 & .313 & .559 & .400 & .687 & .678 & .732 & .419 & .577 & .725 & .634 & .738 & .617 & .383 & .262 & .275 & .366 \\
\hline & 25 & .363 & .360 & .361 & .734 & .748 & 1.098 & 1.048 & .311 & .311 & .557 & .402 & $\begin{array}{l}.689 \\
\end{array}$ & .680 & $\begin{array}{l}.734 \\
\end{array}$ & .418 & .580 & .726 & $\begin{array}{l}.637 \\
\end{array}$ & .743 & 617 & .383 & .257 & .274 & .363 \\
\hline & 26 & .363 & .360 & .361 & .734 & .748 & 1.099 & 1.048 & .310 & .310 & .557 & .402 & .690 & .680 & .734 & .418 & .580 & .726 & $\begin{array}{l}.637 \\
\end{array}$ & .743 & 617 & .383 & .257 & .274 & .363 \\
\hline & 27 & .363 & .360 & .361 & 734 & .748 & 1.099 & 1.048 & .310 & .310 & .557 & .402 & .690 & $\begin{array}{l}.680 \\
\end{array}$ & .734 & .418 & .580 & .726 & $\begin{array}{l}.638 \\
\end{array}$ & .744 & .616 & 384 & 256 & 274 & 362 \\
\hline & 42 & .362 & .358 & .360 & .735 & .754 & 1.121 & 1.058 & .311 & .311 & .557 & .401 & .689 & .679 & .735 & .419 & .581 & .724 & .638 & .745 & .613 & .387 & .255 & 276 & .362 \\
\hline & 59 & .358 & .353 & .356 & .734 & .761 & 1.147 & 1.071 & .313 & .313 & .559 & .400 & .687 & .677 & .734 & .420 & .579 & .722 & .636 & .746 & .607 & .393 & .254 & 278 & .364 \\
\hline \multirow{7}{*}{ 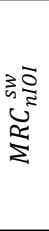 } & 17 & .380 & .369 & .375 & .747 & .733 & 1.071 & 1.035 & .302 & .302 & .550 & .412 & .698 & .684 & .747 & .419 & .596 & .723 & .657 & .772 & .597 & .403 & .228 & .277 & .343 \\
\hline & 20 & .381 & .370 & .375 & .747 & .732 & 1.069 & 1.034 & .302 & .302 & .549 & .413 & .698 & .685 & .747 & .419 & .596 & .724 & .657 & .772 & .598 & .402 & .228 & .276 & .343 \\
\hline & 23 & .381 & .370 & .376 & .747 & .731 & 1.070 & 1.035 & .302 & .302 & .549 & .414 & .698 & .685 & .747 & .419 & .596 & .724 & .657 & .771 & .599 & .401 & .229 & .276 & .343 \\
\hline & 27 & .383 & .372 & .377 & .747 & .732 & 1.072 & 1.035 & .301 & .301 & .549 & .414 & .699 & .686 & $\begin{array}{l}748 \\
\end{array}$ & .418 & .597 & .725 & .658 & .771 & .601 & .399 & .229 & 275 & .342 \\
\hline & 28 & .383 & .372 & .377 & .747 & .732 & 1.073 & 1.036 & .301 & .301 & .549 & .414 & .699 & .686 & .747 & .418 & .596 & .725 & .657 & .770 & .601 & .399 & .230 & .275 & .343 \\
\hline & 30 & .382 & .372 & .377 & .747 & .733 & 1.077 & 1.038 & .301 & .301 & .549 & .414 & .699 & .686 & .747 & .418 & .596 & .725 & .657 & .770 & .602 & .398 & .230 & 275 & 343 \\
\hline & 37 & .380 & .370 & .375 & .746 & .736 & 1.085 & 1.042 & .302 & .302 & .550 & .413 & .698 & .685 & .746 & .419 & .595 & .725 & .656 & .768 & .602 & .398 & .232 & .275 & 344 \\
\hline \multirow{6}{*}{ 它 } & 25 & .388 & .372 & .380 & .751 & .720 & 1.027 & 1.013 & .299 & .299 & .547 & .416 & .701 & .686 & .752 & .420 & .602 & .723 & .665 & .782 & .590 & .410 & .218 & .277 & .335 \\
\hline & 27 & .388 & .373 & .380 & 751 & .719 & 1.025 & 1.012 & .299 & .299 & .547 & .416 & .701 & $\begin{array}{l}.686 \\
\end{array}$ & .752 & .420 & .602 & .723 & .665 & .782 & .591 & .409 & .218 & 277 & .335 \\
\hline & 28 & .388 & .372 & .380 & .751 & .719 & 1.025 & 1.012 & .299 & .299 & .547 & .416 & .701 & $\begin{array}{l}.686 \\
\end{array}$ & .752 & .420 & .602 & .723 & .665 & .782 & .591 & .409 & .218 & .277 & .335 \\
\hline & 35 & .387 & .372 & .379 & .751 & .720 & 1.024 & 1.012 & .299 & .299 & .547 & .415 & .701 & .686 & .751 & .420 & .601 & .723 & .664 & .781 & .591 & .409 & .219 & .277 & .336 \\
\hline & 36 & .386 & .372 & .379 & .750 & .720 & 1.025 & 1.012 & .299 & .299 & .547 & .415 & .701 & .686 & .751 & .420 & .601 & .723 & .663 & .780 & .592 & .408 & .220 & 277 & 337 \\
\hline & 100 & .374 & .364 & .369 & .744 & .727 & 1.035 & 1.017 & .305 & .305 & .552 & .409 & .695 & .682 & .744 & .420 & .592 & .722 & .652 & .767 & .597 & .403 & .233 & 278 & .348 \\
\hline & 9 & .367 & .339 & .353 & .748 & .749 & 1.108 & 1.053 & .310 & .310 & .557 & .407 & .690 & .670 & .750 & .431 & .598 & .703 & .663 & .799 & .540 & .460 & .201 & .297 & .337 \\
\hline & 14 & .378 & .353 & .365 & .752 & .736 & 1.076 & 1.037 & .305 & .305 & .552 & .412 & .695 & $\begin{array}{l}.676 \\
\end{array}$ & .753 & .428 & .602 & .710 & $\begin{array}{l}.668 \\
\end{array}$ & .799 & .554 & .446 & .201 & 290 & 332 \\
\hline & 18 & .379 & .355 & .367 & .752 & .733 & 1.066 & 1.032 & .304 & .304 & .551 & .413 & .696 & .677 & .753 & .427 & .602 & .711 & .668 & .797 & .558 & .442 & .203 & .289 & .332 \\
\hline & 24 & .381 & .359 & .370 & .752 & .729 & 1.056 & 1.028 & .303 & .303 & .550 & .414 & .697 & .679 & .753 & .425 & .602 & .714 & .668 & .794 & .565 & .435 & 206 & 286 & .332 \\
\hline & 31 & .381 & .361 & .371 & .751 & .728 & 1.052 & 1.026 & .303 & .303 & .550 & .414 & .697 & .680 & .752 & .425 & .602 & .715 & .666 & .791 & .569 & .431 & 209 & 285 & .334 \\
\hline & 50 & .381 & .363 & .371 & .750 & .726 & 1.045 & 1.022 & .303 & .303 & .550 & .413 & $\begin{array}{l}.697 \\
\end{array}$ & $\begin{array}{l}.681 \\
\end{array}$ & .751 & .423 & .600 & .717 & .663 & $\begin{array}{l}.785 \\
\end{array}$ & .577 & .423 & .215 & 283 & 337 \\
\hline & 67 & .377 & .361 & .369 & .748 & .727 & 1.043 & 1.021 & 304 & 304 & .551 & .412 & .696 & .681 & .748 & .423 & .597 & .718 & .659 & .780 & .581 & .419 & 220 & 282 & 341 \\
\hline & 99 & .375 & .361 & .368 & .746 & .728 & 1.044 & 1.022 & .305 & .305 & .552 & .410 & .695 & .681 & $\begin{array}{l}.746 \\
\end{array}$ & .422 & .595 & .719 & $\begin{array}{l}.656 \\
\end{array}$ & .775 & .586 & .414 & .225 & 281 & .344 \\
\hline & 100 & .375 & .361 & .368 & .746 & .728 & 1.044 & 1.022 & .305 & .305 & .552 & .410 & .695 & .681 & $\begin{array}{l}.746 \\
\end{array}$ & .422 & .595 & .719 & $\begin{array}{l}.656 \\
\end{array}$ & .775 & .5866 & .414 & .225 & 281 & .344 \\
\hline & 20 & .377 & .368 & .373 & .744 & .727 & 1.034 & 1.017 & .303 & .303 & .551 & .409 & $\begin{array}{l}.697 \\
\end{array}$ & .684 & .745 & .419 & .593 & .724 & .653 & .766 & .602 & .398 & .234 & .276 & 347 \\
\hline & 35 & .379 & .369 & .374 & .746 & .724 & 1.026 & 1.013 & .303 & .303 & .550 & .410 & .697 & $\begin{array}{l}.684 \\
\end{array}$ & .746 & .419 & .594 & .724 & 655 & .768 & 601 & .399 & .232 & 276 & .345 \\
\hline & 36 & .380 & .369 & .374 & .746 & .724 & 1.026 & 1.013 & .302 & .302 & .550 & .411 & .698 & .685 & .746 & .419 & .594 & .724 & .655 & .768 & .601 & .399 & .232 & .276 & .345 \\
\hline & 39 & .380 & .370 & .375 & .746 & .723 & 1.025 & 1.012 & 302 & 302 & .550 & .410 & .698 & .685 & .746 & .419 & .595 & .725 & .655 & .768 & .602 & .398 & 232 & 275 & 345 \\
\hline & 40 & .380 & .370 & .375 & .746 & .723 & 1.025 & 1.012 & .302 & .302 & .550 & .410 & .698 & .685 & .746 & .419 & .595 & .725 & .655 & .768 & .601 & .399 & .232 & 275 & .345 \\
\hline & 18 & .377 & .360 & .368 & .748 & .729 & 1.050 & 1.025 & .304 & .304 & .552 & .412 & .696 & .680 & .749 & .424 & .597 & .717 & .660 & .782 & .578 & .422 & 218 & .283 & .340 \\
\hline & 29 & .379 & .363 & .371 & .748 & .726 & 1.042 & 1.021 & .303 & .303 & .551 & .412 & .697 & .681 & .749 & .422 & .598 & .718 & .661 & .781 & .582 & .418 & 219 & 282 & 339 \\
\hline & 32 & .379 & .364 & .371 & .748 & .726 & 1.041 & 1.020 & .303 & .303 & .550 & .413 & .697 & .682 & .749 & .422 & .598 & .719 & .661 & .781 & .583 & .417 & .219 & 281 & .339 \\
\hline & 36 & .379 & .364 & .372 & $\begin{array}{l}.748 \\
\end{array}$ & .725 & 1.039 & 1.020 & .303 & .303 & .550 & .413 & .697 & .682 & .749 & .422 & .598 & .719 & .660 & .780 & .584 & .416 & .220 & 281 & .340 \\
\hline & 44 & .379 & .364 & .372 & .748 & .725 & 1.038 & 1.019 & .303 & .303 & .550 & .413 & .697 & .682 & .748 & .422 & .597 & .720 & .660 & .779 & .586 & .414 & .221 & 280 & .340 \\
\hline & 45 & .379 & .364 & .372 & .748 & .725 & 1.038 & 1.019 & .303 & .303 & .550 & .413 & .697 & .682 & .748 & .422 & .597 & .720 & .659 & .778 & .586 & .414 & .222 & 280 & .341 \\
\hline & 59 & .377 & .363 & .370 & .747 & .726 & 1.038 & 1.019 & .304 & .304 & .551 & .412 & .696 & .682 & .747 & .422 & .596 & .720 & .657 & .775 & .588 & .412 & .225 & 280 & 343 \\
\hline & 100 & .373 & .361 & .367 & .744 & .729 & 1.042 & 1.021 & .306 & .306 & .553 & .409 & .694 & .681 & .745 & .422 & .593 & .720 & .653 & .770 & .591 & .409 & .230 & 280 & .347 \\
\hline & $\min$ & .353 & .339 & .352 & .728 & .719 & 1.024 & 1.012 & .296 & .296 & .544 & .397 & .684 & 670 & .728 & .415 & .572 & .703 & .628 & .732 & .540 & .374 & .201 & .270 & .332 \\
\hline & max & .393 & .379 & .386 & .753 & .761 & 1.147 & 1.071 & .316 & .316 & .562 & .420 & .704 & $\begin{array}{l}.690 \\
\end{array}$ & .753 & .431 & .604 & .730 & .668 & 799 & .626 & . 460 & 2688 & .297 & .372 \\
\hline
\end{tabular}

The fractional values in the table are displayed based on three significant digits. The heat-map coloring is achieved according to full precision. 


\section{Table 11(on next page)}

Adequate weight-metric combination of the top-performing BNCs: ML1M. 
TABLE 11

Adequate Weight-Metric Combination of THE Top-Performing $B N C_{\mathrm{S}}$ : ML1M

\begin{tabular}{|c|c|c|c|c|c|c|c|c|c|c|c|c|c|c|c|c|c|c|c|c|c|c|c|c|c|}
\hline & & \multicolumn{24}{|c|}{ Performance Metrics } \\
\hline 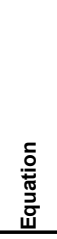 & $\sum_{\infty}^{u}$ & 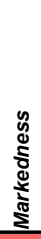 & 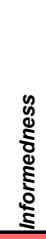 & 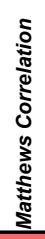 & 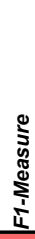 & 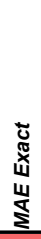 & 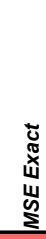 & 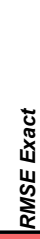 & 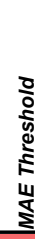 & 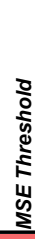 & 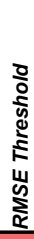 & 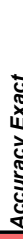 & & & 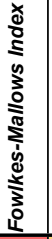 & 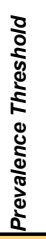 & 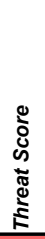 & 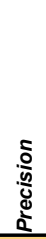 & 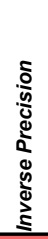 & 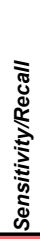 & 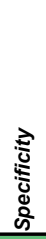 & 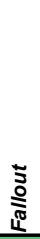 & 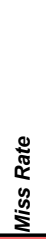 & 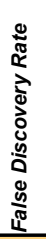 & 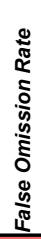 \\
\hline \multirow{6}{*}{ 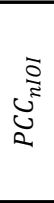 } & 4 & .305 & .308 & .307 & .735 & .797 & 1.200 & 1.098 & .328 & .328 & .572 & 37 & .67 & .6 & .735 & .432 & .581 & .740 & .566 & .730 & .578 & .422 & .270 & .260 & .434 \\
\hline & 31 & .396 & .376 & .386 & .779 & .707 & .981 & .991 & .283 & .283 & .532 & .41 & .71 & .6 & .780 & .422 & .639 & .755 & .641 & .805 & .571 & .429 & .195 & .245 & .359 \\
\hline & 91 & .413 & .382 & .397 & .787 & .696 & .966 & .983 & .27 & .277 & .526 & .42 & .72 & .6 & .788 & .423 & .649 & .754 & .659 & .824 & .559 & .441 & 176 & .246 & .341 \\
\hline & 96 & .412 & .382 & .397 & .787 & .696 & .966 & .983 & .27 & .277 & .526 & .42 & .723 & .6 & .788 & .423 & .649 & .754 & .659 & .824 & .557 & .443 & .176 & .246 & .341 \\
\hline & 99 & .413 & .382 & .397 & .787 & .696 & .966 & .983 & .27 & .277 & .526 & .42 & .72. & .6 & .788 & .423 & .649 & .754 & .659 & 8.825 & .557 & .443 & .175 & .246 & .341 \\
\hline & 100 & .413 & .382 & .397 & .788 & .696 & .966 & .983 & .27 & .277 & .526 & .42 & .72 & .6 & .788 & .423 & .650 & .754 & .659 & .825 & .557 & .443 & .175 & .246 & .341 \\
\hline \multirow{3}{*}{ 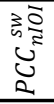 } & 31 & .444 & 368 & .404 & .801 & .688 & .988. & .994 & .270 & .270 & .520 & .44 & .73 & .6 & .804 & .432 & .668 & .740 & .704 & 873 & .495 & .505 & .127 & .260 & 296 \\
\hline & 93 & .439 & .370 & .403 & .799 & .687 & .981 & .990 & .27 & .271 & .520 & .44 & .72 & .6 & .802 & .431 & .666 & .741 & .698 & .868 & .502 & .498 & .132 & .259 & .302 \\
\hline & 100 & .439 & .370 & .403 & .799 & .687 & .979 & .990 & .27 & .271 & .520 & .44 & .72 & .6 & .802 & .431 & .666 & .741 & .698 & .867 & .502 & .498 & .133 & .259 & .302 \\
\hline \multirow{6}{*}{ 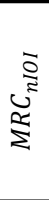 } & 8 & .351 & .344 & .347 & .759 & .747 & 1.075 & 1.03 & .30 & .304 & .552 & .39 & 69 & .6 & .759 & .426 & .611 & .748 & .603 & .769 & .575 & .425 & .231 & .252 & .397 \\
\hline & 48 & .403 & .379 & .391 & .783 & .704 & .980 & .990 & .280 & .280 & .530 & .41 & .720 & .6 & .784 & .422 & .643 & .754 & .649 & .814 & .564 & .436 & .186 & .246 & .351 \\
\hline & 60 & .406 & .379 & .393 & .785 & .702 & .978 & .989 & .274 & .279 & .528 & .41 & .72 & .6 & .785 & .423 & .646 & .754 & .652 & 818 & $\begin{array}{r}.562 \\
\end{array}$ & .438 & .182 & .246 & 348 \\
\hline & 92 & .411 & .380 & .395 & .787 & .701 & .980 & .990 & .278 & .278 & .527 & .42 & .72 & .6 & .787 & .423 & .648 & .753 & .657 & .823 & .557 & .443 & .177 & .247 & .343 \\
\hline & 97 & .411 & .380 & .395 & .787 & .701 & .979 & .990 & .278 & .278 & .527 & .42 & .72 & .6 & $\begin{array}{l}.787 \\
\end{array}$ & .423 & .648 & .753 & .657 & 823 & .557 & .443 & .177 & .247 & 343 \\
\hline & 100 & .410 & .380 & .395 & .787 & .701 & .980 & .990 & .278 & .278 & .527 & .42 & .72 & .6 & .787 & .423 & .648 & .753 & .657 & .823 & .556 & .444 & .177 & .247 & .343 \\
\hline \multirow{7}{*}{ 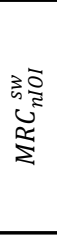 } & 23 & .434 & .364 & .397 & .798 & .698. & 1.001 & 1.00 & .273 & .273 & $\begin{array}{r}.523 \\
\end{array}$ & .43 & .72 & .6 & .800 & .432 & .664 & .739 & .695 & 867 & .497 & .503 & .133 & .261 & .305 \\
\hline & 41 & .434 & .366 & .399 & .798 & 694 & .992 & .996 & .27 & .273 & .522 & .43 & .72 & .6 & .800 & .432 & .664 & .740 & .693 & .865 & .501 & .499 & .135 & .260 & 307 \\
\hline & 44 & .434 & .367 & .400 & .798 & 694 & .992 & .996 & .27 & .272 & .522 & .43 & .72 & .6 & .800 & .431 & .664 & .741 & .694 & .865 & .502 & .498 & .135 & .259 & .306 \\
\hline & 58 & .433 & .368 & .399 & .798 & 694 & 990 & .995 & .27 & .272 & .522 & .43 & .72 & .6 & 800 & .431 & .663 & .741 & .692 & .863 & .505 & .495 & .137 & .259 & .308 \\
\hline & 93 & .431 & .368 & .398 & .797 & 694 & .989. & .995 & .273 & .273 & .522 & .43 & .727 & .6 & .799 & .431 & .662 & .742 & .689 & .861 & .508 & .492 & 139 & .258 & .311 \\
\hline & 94 & .431 & .368 & .398 & .797 & 694 & .989 & .995 & .273 & .273 & .522 & .43 & .727 & .6 & .799 & .431 & .662 & .742 & .689 & 861 & .508 & .492 & .139 & .258 & .311 \\
\hline & 96 & .431 & .368 & .398 & .797 & .694 & .990 & .995 & .27 & .273 & .522 & .43 & .72 & .6 & .799 & .431 & .662 & .742 & .689 & .860 & .508 & .492 & .140 & 258 & .311 \\
\hline \multirow{8}{*}{$\overbrace{}^{\stackrel{?}{2}}$} & 4 & .373 & .346 & .359 & .774 & .736 & 1.070 & 1.03 & .294 & .294 & .542 & .41 & .70 & & .775 & .431 & .631 & .742 & .631 & .809 & .537 & .463 & 191 & .258 & .369 \\
\hline & 13 & .418 & .372 & .394 & .792 & 692 & .969 & .984 & .276 & .276 & .525 & .43 & .72 & & $\begin{array}{l}.793 \\
\end{array}$ & .428 & 655 & .746 & .673 & .843 & .528 & .472 & .157 & .254 & 327 \\
\hline & 36 & .430 & .375 & .402 & .796 & .684 & .951 & .975 & .272 & .272 & .522 & .43 & .72 & & .798 & .428 & .661 & .745 & .685 & 854 & .520 & .480 & .146 & .255 & .315 \\
\hline & 41 & .431 & .374 & .402 & .796 & .683 & .950. & .975 & .272 & .272 & .522 & .43 & .72 & .6 & .798 & .428 & .661 & .745 & .686 & .855 & .520 & .480 & .145 & .255 & .314 \\
\hline & 45 & .430 & .374 & .401 & .796 & .683 & .949 & .974 & .272 & .272 & .522 & .43 & .72 & .6 & .798 & .429 & .661 & .745 & .685 & 855 & .519 & .481 & .145 & .255 & .315 \\
\hline & 50 & .430 & .374 & .401 & .796 & .683 & .951 & .975 & .272 & .272 & .522 & .43 & .72 & .6 & .798 & .429 & .661 & .745 & .686 & .855 & .518 & .482 & .145 & .255 & .314 \\
\hline & 72 & .429 & .372 & .399 & .796 & 684 & .954 & .977 & .27 & .273 & .522 & .43 & .72 & .6 & .798 & .429 & .661 & .744 & .685 & .855 & .517 & .483 & .145 & .256 & .315 \\
\hline & 83 & .429 & .371 & .399 & .796 & .684 & .954 & .977 & .277 & .273 & .523 & .43 & .72 & .6 & .798 & .429 & .661 & .744 & .685 & 855 & .516 & .484 & .145 & .256 & .315 \\
\hline \multirow{8}{*}{ 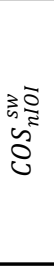 } & 18 & .428 & .343 & .383 & .797 & .712 & 1.044 & 1.022 & .278 & .278 & .527 & .43 & .72 & .6 & .800 & .438 & .662 & .730 & .699 & .878. & .466 & .534 & .122 & .270 & .301 \\
\hline & 30 & .430 & .347 & .387 & .797 & . 706 & 1.030 & 1.01 & .27 & .277 & .526 & .43 & .72 & .6 & .801 & .437 & .663 & .731 & .699 & 877 & .470 & .530 & .233 & .269 & 301 \\
\hline & 72 & .429 & .352 & .389 & .797 & .701 & 1.015 & 1.00 & .276 & .276 & .526. & .43 & .72 & .6 & .800 & .436 & .662 & .733 & .696 & $\begin{array}{r}.873 \\
\end{array}$ & .479 & .521 & .127 & .267 & 304 \\
\hline & 91 & .428 & .352 & .388 & .796 & .701 & 1.012 & 1.000 & .277 & .277 & .526 & .43 & .72 & .6 & .799 & .436 & .662 & .734 & .694 & 871 & .481 & .519 & .129 & .266 & .306 \\
\hline & 92 & .428 & .352 & .388 & .7966 & .701 & 1.012 & 1.006 & .27 & .277 & .526 & .43 & .72 & & .799 & .436 & .662 & .734 & .694 & .871 & .482 & .518 & .129 & .266 & .306 \\
\hline & 97 & .427 & .352 & .388 & .796 & .701 & 1.011 & 1.000 & .27 & .277 & .526 & .43 & .72 & & .799 & .436 & .661 & .734 & .693 & 870.870 & .482 & .518 & .130 & .266 & .307 \\
\hline & 99 & .427 & .352 & .388 & .796 & .701 & 1.011 & 1.00 & .277 & .277 & .526 & .43 & .72 & & .799 & .435 & .661 & .734 & .693 & .870 & .482 & .518 & 130 & .266 & .307 \\
\hline & 100 & .427 & .352 & .388 & .796 & .701 & 1.011 & 1.00 & .27 & .277 & .526 & .43 & .72 & & .799 & .436 & .661 & .734 & .693 & 870 & .482 & .518 & .130 & .266 & .307 \\
\hline \multirow{7}{*}{ 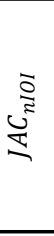 } & 10 & .396 & .369 & .382 & .781 & .705 & .983 & .991 & .284 & .284 & .533 & .41 & .71 & & .782 & .425 & .641 & .750 & .646 & 814 & .555 & .445 & .186 & .250 & .354 \\
\hline & 29 & .417 & .382 & .399 & .790 & 689 & .947 & .973 & .274 & .275 & .524 & .42 & .72 & .6 & .791 & .424 & .652 & .752 & .665 & .831 & .551 & .449 & .169 & .248 & .335 \\
\hline & 53 & .420 & .382 & .400 & .791 & .687 & .942 & .971 & .274 & .274 & .524 & .42 & .72 & .6 & .792 & .424 & .654 & .752 & .668 & .834 & .548 & .452 & .166 & .248 & .332 \\
\hline & 58 & .420 & .382 & .400 & .791 & .687 & .943 & .971 & .274 & .274 & .524 & .42 & .72 & & .792 & .424 & .654 & .752 & .668 & .834 & .547 & .453 & .166 & .248 & .332 \\
\hline & 84 & .420 & .381 & .400 & .791 & .688 & .944 & .972 & .274 & .274 & .524 & .42 & .72 & & .792 & .424 & .654 & .751 & .668 & .835 & .546 & .454 & .165 & .249 & .332 \\
\hline & 90 & .419 & .380 & .399 & .791 & .688 & .945 & .972 & .277 & .275 & .524 & .42 & .72 & & .792 & .425 & .654 & .751 & .668 & 835 & .545 & .455 & .165 & .249 & .332 \\
\hline & 99 & .419 & .380 & .399 & .791 & .688 & .945 & .972 & .277 & .275 & .524 & .42 & .72 & & .792 & .425 & .654 & .751 & .668 & .836 & .544 & .456 & .164 & .249 & .332 \\
\hline & 4 & .366 & .333 & .349 & .773 & .745 & 1.098 & 1.048 & .297 & .297 & .545 & .40 & .70 & & .774 & .435 & .630 & .735 & .631 & .815 & .518 & .482 & .185 & .265 & .369 \\
\hline & 25 & .422 & .366 & .393 & .794 & 692 & .972 & .986 & .276 & .276 & .525 & .43 & .72 & .6 & .795 & .431 & .658 & .742 & .680 & .853 & .513 & .487 & 147 & .258 & .320 \\
\hline $5=$ & 54 & .425 & .366 & .395 & .795 & .688 & .965 & .982 & .27 & .275 & .524 & .43 & .72 & .6 & .797 & .431 & .659 & .742 & .683 & .856 & .510 & .490 & .144 & .258 & .317 \\
\hline$\pi$ & 55 & .425 & .366 & .394 & .795 & .688 & .965 & .982 & .275 & .275 & .524 & .43 & .72 & & .797 & .431 & .659 & .742 & .683 & .856 & .510 & .490 & 144 & .258 & .317 \\
\hline & 56 & .425 & .366 & .395 & .795 & .688 & .965 & .982 & .279 & .275 & .524 & .43 & .72 & & .797 & .431 & $\begin{array}{l}.659 \\
\end{array}$ & .742 & .683 & .856 & .510 & .490 & .144 & .258 & .317 \\
\hline & 75 & .425 & .366 & .394 & .795 & .689 & .966 & .983 & .275 & .275 & .524 & .43 & .72 & .6 & .797 & .431 & .659 & .741 & .683 & .856 & .509 & .491 & .144 & .259 & 317 \\
\hline & $\min$ & .305 & .308 & .307 & .735 & .683. & .942 & 971 & .270 & .270 & .520 & .37 & .67 & & .735 & .422 & .581 & .730 & .566 & .730 & .466 & .422 & 122 & .245 & 296 \\
\hline & $\max$ & . 444 & .382 & .404 & .801 & .797 & 1.200 & 1.09 & .328 & .328 & .572 & .44 & .73 & & .804 & .438 & .668 & .755 & .704 & .878 & .578 & .534 & .270 & .270 & .434. \\
\hline
\end{tabular}

The fractional values in the table are displayed based on three significant digits. The heat-map coloring is achieved according to full precision. 


\section{Box 1 (on next page)}

Algorithm 1: Pseudocode of the experimental process for an individual test package. 


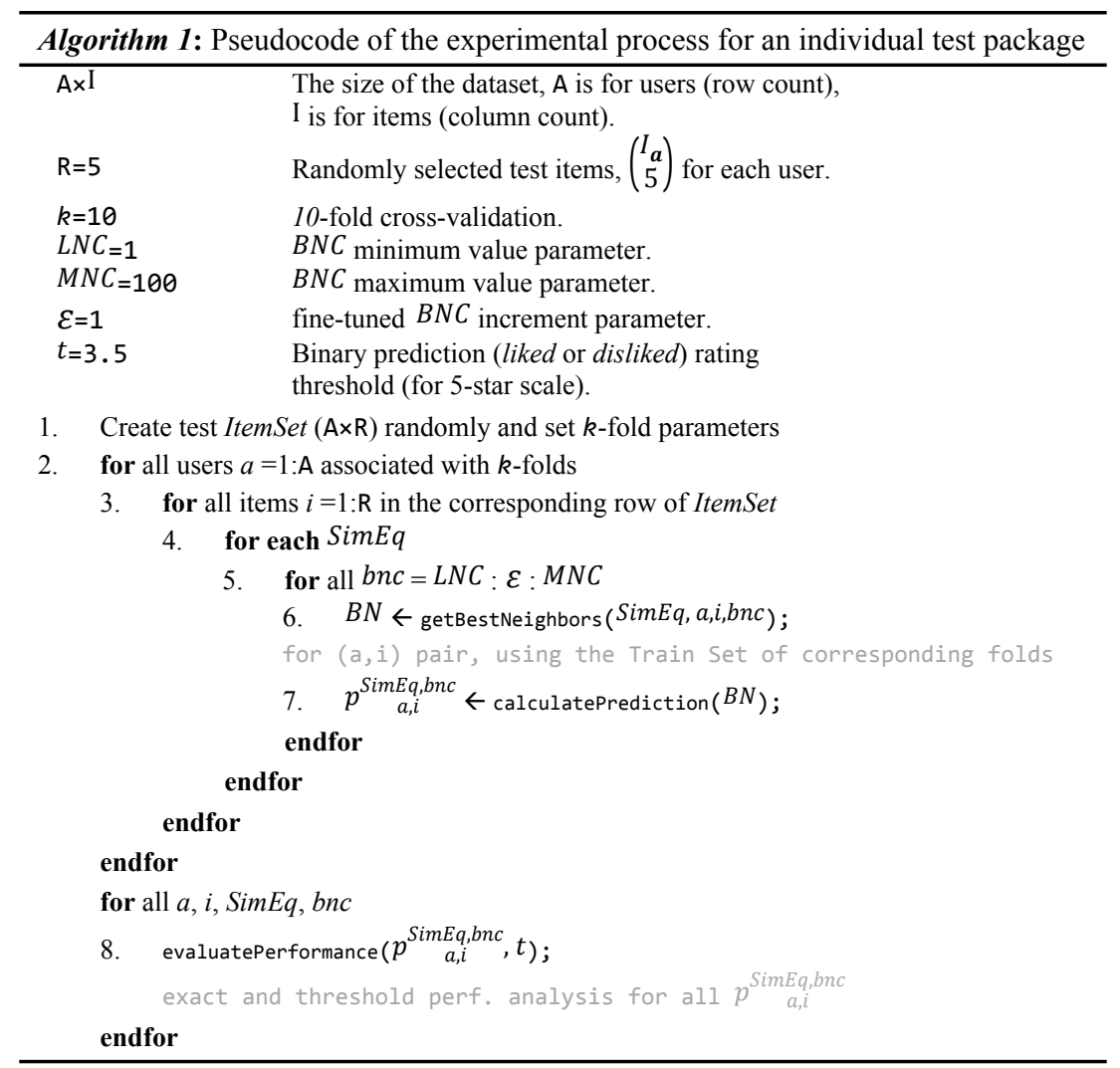

